\author{
Universidade de São Paulo \\ Instituto de Astronomia, Geofísica e Ciências Atmosféricas \\ Departament of Astronomy
}

Henrique Lupinari

\title{
Testing G-CLEF Fiber Efficiency: the Impact of using Adaptive Optics
}

São Paulo 



\author{
Henrique Lupinari
}

\title{
Testing G-CLEF Fiber Efficiency: the Impact of using Adaptive Optics
}

Thesis presented to the Department of Astronomy of Instituto de Astronomia, Geofísica e Ciências Atmosféricas of Universidade de São Paulo as a parcial requirement to obtain a Master of Sciences degree.

Field of Research: Astronomy

Advisor: Prof. Dr. Claudia Mendes de Oliveira

São Paulo 

I dedicate this thesis to my family and closest friends, who gave me the support I needed throughout the course of this work! 



\section{Acknowledgements}

To Heloísa, Guilherme and Isabella, my siblings, and Andréa and Carlos, my parents;

To my advisor Claudia Mendes de Oliveira for the support in all aspects;

To all my friends, who supported me during the last two years (and much more than that);

To Andrew Szentgyorgyi, Sagi Ben-Ami, Steve Eikenberry, Fábio Cafardo and André Vitorelli for playing important roles in this work;

To CAPES, for financing my scholarship;

To FAPESP, through the GMT Brazil Office, for financial support of several activities related to my dissertation work, under project 2011/51680-6;

To IAG/USP for the infrastructure provided;

To Harvard-Smithsonian Center for Astrophysics for receiving and financing me during a two-months visit;

Esta tese/dissertação foi escrita em ATEX com a classe IAGTESE, para teses e dissertações do IAG. 

"The path of the stars is as reliable as the instruments that measure them."

Scholar of Stars - Magic: the Gathering 



\section{Abstract}

G-CLEF is a state of the art echelle spectrograph for the Giant Magellan Telescope (GMT), expected to be completed for first light. Its good performance for seeing limited observations may be further improved with the future use of an extreme Adaptive Optics (AO) system to cancel out atmospheric disturbances and optimize throughput. The goal of our project is to perform laboratory optical-bench experiments to simulate G-CLEF working with and without AO. We measure the efficiency of the G-CLEF fiber in both cases to compute the gain in using an AO system. 



\section{Resumo}

O G-CLEF é um espectrógrafo echelle de última geração para o Telescópio Gigante Magalhães (GMT), com previsão de conclusão para a primeira luz. Seu bom desempenho em observações limitadas pelo seeing pode ser aprimorado ainda mais com o uso futuro de um sistema de Óptica Adaptativa (AO) para cancelar perturbações atmosféricas e otimizar seu rendimento. O objetivo do nosso projeto é realizar experimentos de bancada óptica no laboratório para simular o G-CLEF trabalhando com e sem AO. Medimos a eficiência da fibra do G-CLEF nos dois casos para calcular o ganho gerado pelo uso de um sistema de $\mathrm{AO}$. 



\section{List of Figures}

1.1 Motion in the host star due to a second body sharing the system with it and the reflex on Doppler effect of spectral lines. (Image credit: ESO 2007)

1.2 Precision required to detect a planet around a star through precision radial velocity measurements, in therms of spectral type of host star and period of planetary orbit. Planets shown here where detected through transit observations. Red and blue bands are the habitable zones of the host star. (Image credit: Chester Harman) . . . . . . . . . . . . . . .

1.3 G-CLEF residing in the Azimuth Platform of GMT. Connection between instrument and telescope focus is done through optical fibers. Figure 1 from Szentgyorgyi et al. (2014) . . . . . . . . . . . . . . . .

1.4 Spectrograph optical design. Dashed line shows the zero order (light that was not deviated by the VPH). Solid line shows light path throughout the spectrograph system. Figure 4 from Szentgyorgyi et al. (2016). . . . . . . .

1.5 Fiber-fed system: each GMT mirror segment will feed a single $100 \mu \mathrm{m}$ fiber on PRV and NS-PRV mode. Figure 5 from Szentgyorgyi et al. (2018). . . .

1.6 Pupil slice allows to reorganize the fibers, from the shape of GMT's pupil $(\sim 300 \mu \mathrm{m}$ in diameter $)$ to a slit with width of $100 \mu \mathrm{m} . \ldots . . . . . .31$

1.7 Wavefront being disrupted by atmospheric turbulence. . . . . . . . . . . 32

1.8 Oversimplified scheme of disrupted wavefront and Fried's coherence length,

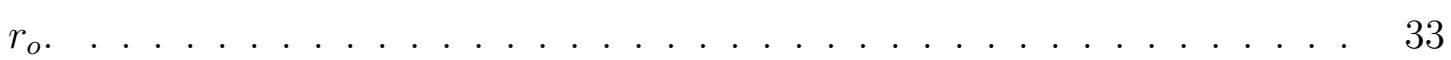

1.9 Schematics of adaptive optics system. . . . . . . . . . . . . . . 34

1.10 Very Large Telescope's (VLT) UT4 using sodium lasers to enable AO cor-

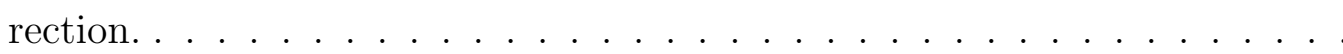


1.11 Point-spread-function of three scenarios: diffraction limited case, AO corrected and seeing limited. . . . . . . . . . . . . . . . . . 36

1.12 Phase screen: interface between polymers of slightly different indices of refraction. Figure from Lexitek Inc.. . . . . . . . . . . . . . . . 37

1.13 Left: Seeing phase screen; Right: AO corrected phase screen. . . . . . . . . 38

1.14 Optical fiber structure. . . . . . . . . . . . . . . . . . 38

1.15 Optical fiber FDR scheme. . . . . . . . . . . . . . . . . . . . 40

1.16 Ray traces through double scrambler lens. Figure 12 of Halverson et al.

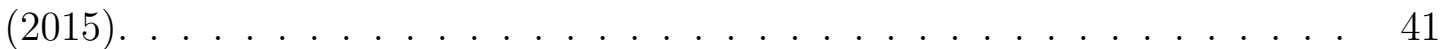

2.1 Variation in encircled energy over time for an uncorrected wavefront as a function of wavelength: $440 \mu \mathrm{m}$ (blue), $550 \mu \mathrm{m}$ (green), $640 \mu \mathrm{m}$ (yellow) and $790 \mu \mathrm{m}(\mathrm{red})$.

2.2 Variation in encircled energy over time for an $\mathrm{AO}$ corrected wavefront as a function of wavelength: $440 \mu \mathrm{m}$ (blue), $550 \mu \mathrm{m}$ (green), $640 \mu \mathrm{m}$ (yellow) and $790 \mu \mathrm{m}$ (red).

3.1 Illustration of misalignment effects on fiber's far field illumination. Dashed lines represents the light rays that does not enter the optical fiber. Top: Seeing disk aligned with fiber's optical axis, yielding a homogeneous far field illumination. Bottom: Seeing disk misaligned with fiber's optical axis, yielding a annulus pattern in the far field illumination. Figure 1 from Mello et al. (2018) . . . . . . . . . . . . . . . . . . . . . 48

3.2 Annulus pattern in far field illumination yielded by a misaligned fiber in the lab. Experiment made in IAG lab.

3.3 Standard deviation of encircled energy varying with turbulence strength. Dashed line represents the simulation without adaptive optics while continuum line the ones with AO correction with 6 and 12 mag guidestars. Right:

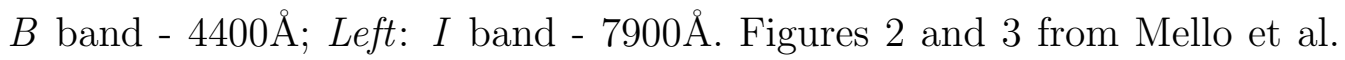
(2018).

3.4 Fiber throughput power varying with time when subjected to AO correction (green line) and no correction (red line). 
4.1 Schematics of optical bench setup; Top: Mirrors facing each other, allowing beam to transfer directly from first to second relay; Bottom: Mirrors facing the fiber ends, forcing the beam to go through the fiber in between relays.

4.2 Picture of the setup used in the experiment. . . . . . . . . . . . . 56

4.3 Top: Raw data of one set of measurement for FRD experiment. Bottom: Zoom in to show data of interest. . . . . . . . . . . . . . . 59

4.4 Example of raw data of power vs time for throughput and stability measu-

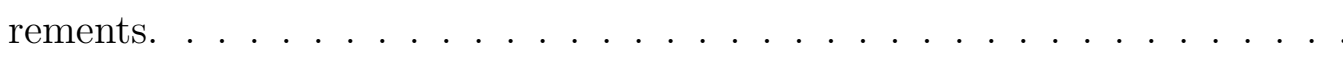

5.1 Dataset for our FRD experiment: shown in green dots are the time coordinates of the starting points of useful data and in red squares the coordinates for the ends. . . . . . . . . . . . . . . . . . . . 62

5.2 Focal ratio profile of the beam in three different environments with fiber. . 63

5.3 Power over time of each of the six configurations and noise curve. Raw data. 65

5.4 Normalized throughput over time for each of the six configurations. . . . . 65

5.5 Fourier transform of the data taken using the three configurations that use the optical fiber. . . . . . . . . . . . . . . . . 66

A.1 Input fiber surface of 'Fiber 1', cleaved with LCC cleaver. This fiber was not used. . . . . . . . . . . . . . . . . .

A.2 Output fiber surface of 'Fiber 1', cleaved with LCC cleaver. This fiber was not used. . . . . . . . . . . . . . . . . . .

A.3 Input fiber surface of 'Fiber 2', cleaved with common, commercial cleaver. This was the fiber used. . . . . . . . . . . . . . . . .

A.4 Output fiber surface of 'Fiber 2', cleaved with common, commercial cleaver. This was the fiber used. 



\section{List of Tables}

1.1 Required sensitivity to detect a planet around a star through radial velocity measurements. [1] Currently achievable precision. [2] Precision expected to be achieved by the time of G-CLEF's first light. [3] G-CLEF specification precision. [4] G-CLEF goal precision. (Szentgyorgyi et al., 2014) . . . . . . 24

1.2 Comparison of the three ELTs. (private communication, Bernstein) . . . . 26

1.3 G-CLEF's observation modes and its parameters. Throughput requirements at 650nm, as described in Podgorski (2018) throughput error budget report. 29

2.1700 mas encircled energy as a function of wavelength and wavefront correction strategy. . . . . . . . . . . . . . . . . . . . . 44

2.2 Variability in 700 mas encircled energy as a function of wavelength and wavefront correction strategy. . . . . . . . . . . . . . .

2.3 700 mas encircled energy as a function of seeing and wavefront correction strategy. The wave length is $550 \mu \mathrm{m} \ldots \ldots \ldots$. . . . . . . . . .

3.1 Average fiber throughput depending on whether the observation is AO corrected or not. . . . . . . . . . . . . . . . . 50

4.1 Lenses used in the setup. . . . . . . . . . . . . . . . . . . 56

5.1 Information of throughput and stability for each of the six configurations; ploted in figure $5.3 \ldots \ldots \ldots$. . . . . . . . . . . . . . . . 64

5.2 Normalized stability and throughput for each of the six configurations. . . 66

5.3 Information on stability and throughput after taking into account the presence of an intrinsic beating in the setup. . . . . . . . . . . . . . . . 67 



\section{Contents}

1. Introduction . . . . . . . . . . . . . . . . . . . . 21

1.1 Exoplanetary Science . . . . . . . . . . . . . . . . . . . 21

1.1.1 Detection technique: Doppler Radial Velocity . . . . . . . . . 22

1.2 G-CLEF . . . . . . . . . . . . . . . . 25

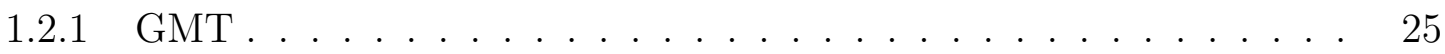

1.2 .2 G-CLEF Overview . . . . . . . . . . . . . . . . 26

1.2.3 Observation Modes . . . . . . . . . . . . . . . . . . . . . 28

1.2.4 Optical System - Spectrograph, Cameras and Detectors . . . . . . . 28

1.2.5 Fiber-feed System for Precision Radial Velocity Mode . . . . . . . . 30

1.3 Adaptive Optics . . . . . . . . . . . . . . . . . . . . . . . . . 31

1.3.1 Lexitec Phase Screens . . . . . . . . . . . . . . . . . 37

1.4 Optical Fibers . . . . . . . . . . . . . . . . . . . . 38

1.4 .1 Effects on Beam . . . . . . . . . . . . . . . . . . . 39

1.4.2 Double Scrambler . . . . . . . . . . . . . . . . . . . . . 40

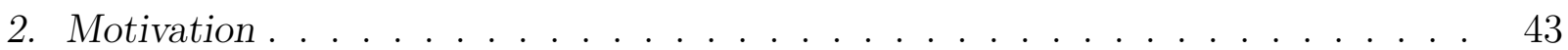

3. Application of Adaptive Optics for Illumination Stability in Precision Radial Velocity Measurements in Astronomical Spectroscopy . . . . . . . . . . . . . . . . . 47

3.1 Illumination Stability Effects on PRV Measurements . . . . . . . . . . . 47

3.2 Adaptive Optics Stability Simulations . . . . . . . . . . . . . . . . . . 49

3.3 Optical Bench Experiment . . . . . . . . . . . . . . . 50

3.4 Conclusion . . . . . . . . . . . . . . . . . . 51 
4. Optical Bench Experiments . . . . . . . . . . . . . . . . . . . . 53

4.1 Optical Bench Setup . . . . . . . . . . . . . . . . 53

4.1.1 Fiber Cleaving and Characterization Techniques . . . . . . . . 56

4.2 Measurements . . . . . . . . . . . . . . . . . . 57

4.2 .1 Focal Ratio Degradation . . . . . . . . . . . . . . . . . 58

4.2 .2 Throughput and Stability . . . . . . . . . . . . . 59

5. Analysis and Results . . . . . . . . . . . . . . . . . . . 61

5.1 Focal Ratio Degradation . . . . . . . . . . . . . . . . 61

5.2 Throughput and Stability . . . . . . . . . . . . . . 63

6. Discussion, Conclusion and Prospects . . . . . . . . . . . . . . 69

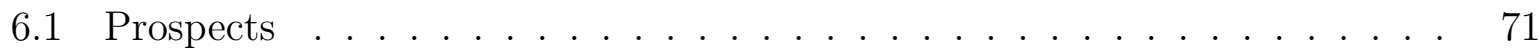

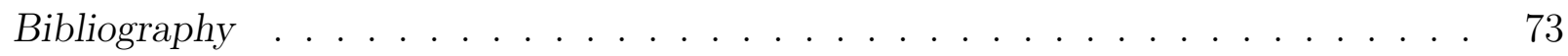

$\begin{array}{ll}\text { Appendix } & 77\end{array}$

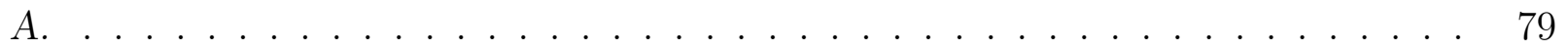


Chapter 1

\section{Introduction}

This chapter will address a brief introduction to topics that are relevant for the complete understating of this thesis. The main goal of this dissertation is to study the effects that adaptive optics may have on the efficiency of a fiber feed system, more specifically on the optical fibers of G-CLEF, a GMT high resolution spectrograph designed to search for and characterize exoplanets similar to Earth. Section 1.1 brings a short overview on exoplanetary science, section 1.2 contains a description of G-CLEF, the instrument studied in this work, together with a brief introduction on adaptive optics and optical fibers in sections 1.3 and 1.4 , respectively.

\subsection{Exoplanetary Science}

Ever since human kind realized we are not the center of the universe, questions naturally came to mind: are we alone in the universe? Is there life somewhere else in space? These are two of the most ancient philosophical and existential questions humans face. This longing has always lived in the imaginary of humanity, and it shows in artistic expressions such as literature in Bradbury and Vonnegut, for example, and in numerous movies. It is in Science's best interest to respond to this longings.

It was only in the past couple of decades that we verified the existence of planets orbiting other stars, and that we realized that every star in our galaxy could host its own planetary system. In order for a body to be defined as a planet, according to the International Astronomical Union (IAU), it has to meet some criteria: (1) orbit a star, (2) have enough mass to reach hydrostatic equilibrium, (3) be the prominent body on its orbit and (4) have mass below the thermonuclear fusion of deuterium limit $\left(\sim 13 M_{\text {Jupiter }}\right)$. If 
it does not meet the last criterion it is no longer considered a planet, but a brown dwarf (Boss et al., 2007).

The first exoplanet was detected around a milisecond pulsar (Wolszczan and Frail, 1992). The first confirmed exoplanet around a main sequence star was $51 \mathrm{Peg}$ b, a gas giant orbiting a G star on a multiple system (Mayor and Queloz, 1995).

Recent studies show that $62 \%$ of the stars in our galaxy host at least one Earth-mass planet, 52\% Neptune-mass and 17\% Jupiter-mass planets (Cassan et al., 2012). It is already acknowledged that rocky, Earth-size exoplants are common (Weiberg et al., 2018). What is still to be studied is the nature of their atmospheres. Ranging from a Venuslike atmosphere to no atmosphere at all, not to mention the vast amount of different non-imagined atmospheres, only a narrow and specific set of atmospheric characteristics is expected to host life. As expected, common agreement is that, most probably, life as we know it could be hosted only in planets similar to Earth: rocky and in the habitable zone, i.e. at a certain distance from the host star in which it is able to maintain water in liquid state, and therefore, maintain surface temperature at around 300K. Such type of exoplanets was already found. For example, TRAPPIST-1 system is a seven terrestrial planet system around a dwarf star in which all of the planets present favorable conditions to contain liquid water and therefore harbour life (Gillon et al., 2017). Proxima Centauri, our closest stellar neighbour, hosts a planet in the habitable zone too (Anglada-Escudé et al., 2016).

The prime goal of exoplanetary science is to understand how such a variety of planetary systems form and develop, and one of many interesting questions of the field is whether or not some of them have the conditions to incubate life.

\subsubsection{Detection technique: Doppler Radial Velocity}

There are several methods to detect exoplanets: transit, Doppler radial velocitiy measurements, direct imaging, microlensing and a few others. The vast majority of exoplanets so far was detected through transit, the main contribution coming from Kepler survey. The second most prominent method of detection is radial velocity (RV). Thus far, there have been 4034 exoplanets confirmed (NASA Exoplanet Archive; as for August 2019), among them 3130 through transit and 762 through RV. Although transit is a more efficient way of detecting exoplanets, it only tell us the radius of the planet and its orbital period. Whereas 
when combining information from transit and radial velocity measurements we can infer the planet's mass and therefore its density.

Doppler radial velocity measurement works, as the name suggests, detecting shifts in the star spectral lines caused by reflex motion of the star, which is caused by the presence of an orbiting planet. A massive body orbiting the host star will cause it to move around the center of mass of the system and this movement will reflect as Doppler shifts on the spectral lines (depending on the observer vantage point), as can be seen in figure 1.1. The magnitude of the movement depends on the mass of both the host star and the planet, and the distance between them. In table 1.1 is shown the reflex movement of the host star in numerous situations, and the sensitivity necessary to detect them, which is also shown, more graphically, in figure 1.2 .

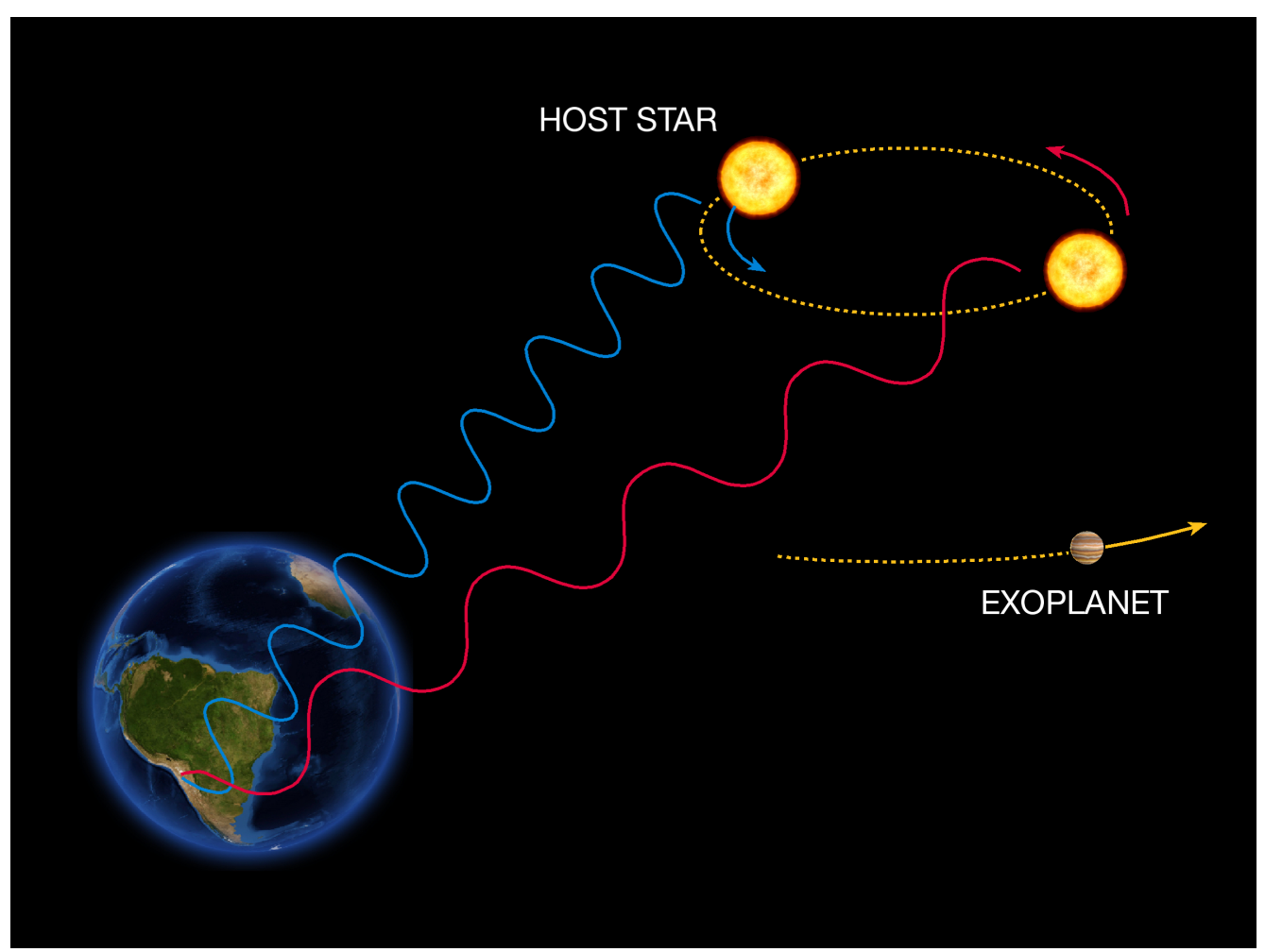

Figure 1.1: Motion in the host star due to a second body sharing the system with it and the reflex on Doppler effect of spectral lines. (Image credit: ESO 2007)

Currently, we are only able to detect a motion of the order of $50 \mathrm{~cm} / \mathrm{s}$. In order to detect an Earth-like planet orbiting a Solar-like star, the instrument needs to be able to detect stellar motion of the order of $10 \mathrm{~cm} / \mathrm{s}$. G-CLEF is designed to achieve that precision. 


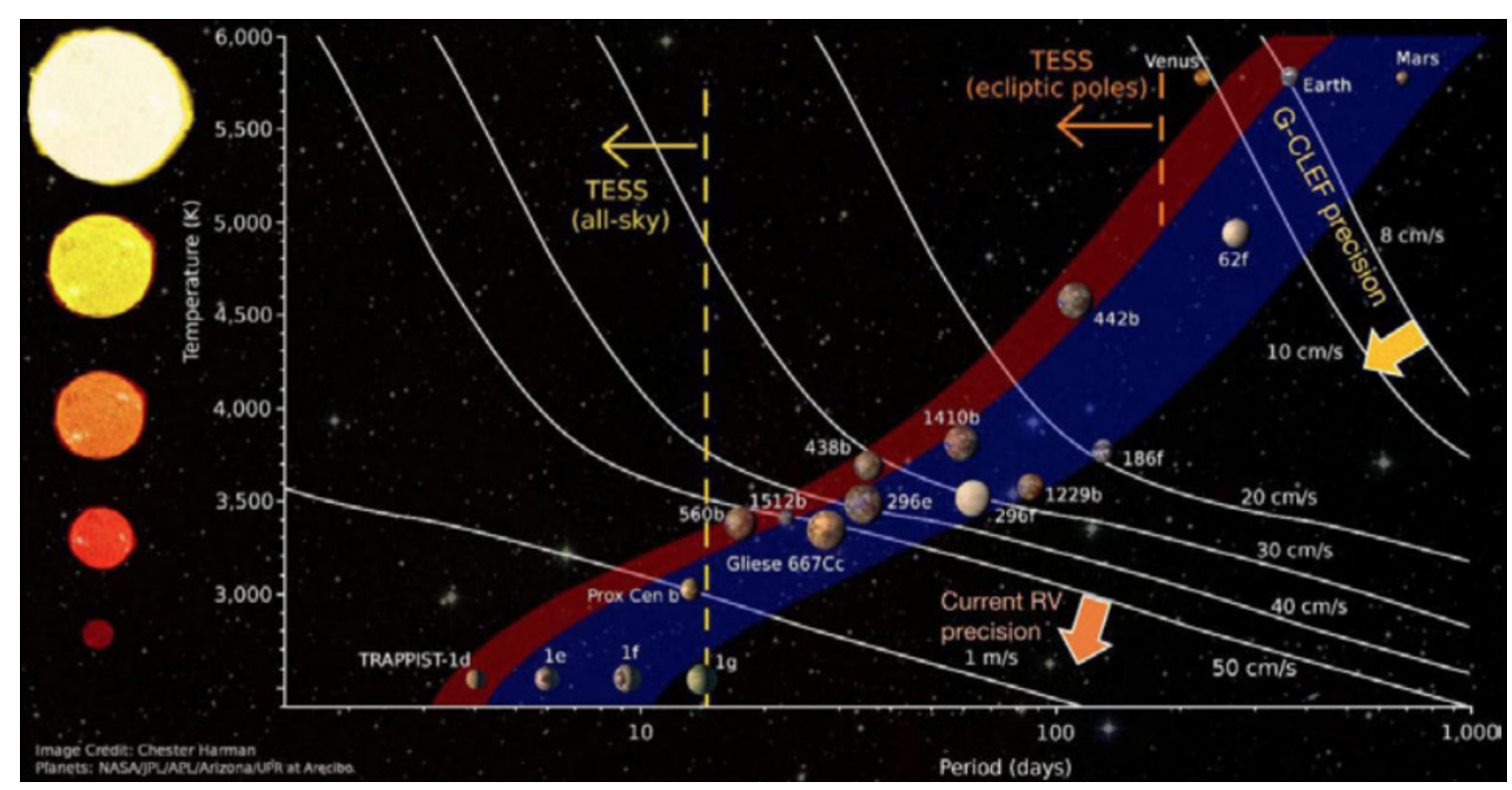

Figure 1.2: Precision required to detect a planet around a star through precision radial velocity measurements, in therms of spectral type of host star and period of planetary orbit. Planets shown here where detected through transit observations. Red and blue bands are the habitable zones of the host star. (Image credit: Chester Harman)

Table 1.1 - Required sensitivity to detect a planet around a star through radial velocity measurements. [1] Currently achievable precision. [2] Precision expected to be achieved by the time of G-CLEF's first light. [3] G-CLEF specification precision. [4] G-CLEF goal precision. (Szentgyorgyi et al., 2014)

\begin{tabular}{|c|c|c|c|c|c|c|}
\hline \multirow[t]{2}{*}{ Planet Mass } & \multirow{2}{*}{$\begin{array}{c}\text { Orbit } \\
\text { Radius (AU) }\end{array}$} & \multicolumn{5}{|c|}{ Required Reflex Velocity Sensitivity (m/s) } \\
\hline & & $\mathrm{G} 2 \mathrm{~V}$ & M0V & $\mathrm{M} 2 \mathrm{~V}$ & $\mathrm{M} 4 \mathrm{~V}$ & $\mathrm{M} 6 \mathrm{~V}$ \\
\hline Jupiter $\left(318 M_{\text {Earth }}\right)$ & 0.1 & $89.8^{[1]}$ & $116^{[1]}$ & $136^{[1]}$ & $201^{[1]}$ & $284^{[1]}$ \\
\hline Jupiter $\left(318 M_{\text {Earth }}\right)$ & 1.0 & $28.4^{[1]}$ & $36.7^{[1]}$ & $42.9^{[1]}$ & $63.6^{[1]}$ & $89.9^{[1]}$ \\
\hline Jupiter $\left(318 M_{\text {Earth }}\right)$ & 5.0 & $12.7^{[1]}$ & $16.4^{[1]}$ & $19.1^{[1]}$ & $28.4^{[1]}$ & $40.2^{[1]}$ \\
\hline Neptune $\left(17 M_{\text {Earth }}\right)$ & 0.1 & $4.8^{[1]}$ & $6.2^{[1]}$ & $7.2^{[1]}$ & $10.8^{[1]}$ & $15.2^{[1]}$ \\
\hline Neptune $\left(17 M_{\text {Earth }}\right)$ & 1.0 & $1.5^{[1]}$ & $2.0^{[1]}$ & $2.3^{[1]}$ & $3.4^{[1]}$ & $4.8^{[1]}$ \\
\hline Super Earth $\left(5 M_{\text {Earth }}\right)$ & 0.1 & $1.4^{[1]}$ & $1.8^{[1]}$ & $2.1^{[1]}$ & $3.1^{[1]}$ & $4.4^{[1]}$ \\
\hline Super Earth $\left(5 M_{\text {Earth }}\right)$ & 1.0 & $0.45^{[3]}$ & $0.57^{[2]}$ & $0.67^{[1]}$ & $1.0^{[1]}$ & $1.4^{[1]}$ \\
\hline Earth & 0.1 & $0.28^{[3]}$ & $0.37^{[3]}$ & $0.43^{[3]}$ & $0.68^{[2]}$ & $0.89^{[2]}$ \\
\hline Earth & 1 & $0.09^{[3]}$ & $0.12^{[3]}$ & $0.13^{[3]}$ & $0.20^{[3]}$ & $0.28^{[3]}$ \\
\hline $\operatorname{Mars}\left(0.11 M_{\text {Earth }}\right)$ & 0.1 & 0.03 & $0.04^{[4]}$ & $0.05^{[4]}$ & $0.07^{[4]}$ & $0.09^{[3]}$ \\
\hline Mars $\left(0.11 M_{\text {Earth }}\right)$ & 1.0 & 0.009 & 0.012 & 0.014 & 0.021 & 0.030 \\
\hline
\end{tabular}




\section{$1.2 \quad G-C L E F$}

\subsubsection{GMT}

The Giant Magellan Telescope (GMT) is a $24.5 m$ telescope in construction at Cerro Las Campanas, Chile. It will be one of the three new generation ground-based "extremely large telescopes" (ELTs), responsible for the next great leap on our understanding in Astrophysics. Its programs will address some of the most intriguing and current inquiries in Astronomy nowadays, such as the many aspects of the origin of life in the universe, for instance studying Exo-Earths orbiting a Solar twin star and investigating the nature of dark energy and black holes. In addition, and perhaps more exciting, we will discover things that we cannot predict yet.

The GMTO Corporation is a consortium of universities and research institutes around the world, a non-profit organization in charge of design, construction and operation of GMT. The GMT consortium is composed of the following partners: Arizona State University, Astronomy Australia Ltd., the Australian National University, the Carnegie Institution for Science, the São Paulo Research Foundation (FAPESP), Harvard University, Korea Astronomy and Space Science Institute, Smithsonian Intuition, Texas A\&M University, The University of Texas at Austin, University of Arizona, University of Chicago and University of Chile

The Las Campanas Observatory is located at the southern part of Chilean Atacama Desert. It is also home to other telescopes, such as the Magellan Telescopes, two $6.5 \mathrm{~m}$ telescopes. The site was chosen due to the absence of any present and future significant major threats regarding light pollution and also its outstanding weather conditions; at an altitude of $2,550 \mathrm{~m}$, it shows median seeing of 0.62 arcsec full width at half maximum (FWHM) at $550 \mathrm{~nm}$.

The chosen design for GMT is an Aplanatic Gregorian. Besides being a considerably less compact design, this kind of mount offers several advantages, for example: since the beam comes to a focus before reaching the secondary mirror, it allow us to have in-telescope calibration of adaptive optics with an artificial star at prime focus. The difference between Aplanatic and Classical Gregorian design is that on the first, both, primary and secondary mirrors, are concave ellipsoidal shaped, while on the latter the primary is parabolic. GMT's primary mirror is segmented in seven mirrors, each $8.4 \mathrm{~m}$ in 
diameter. Each of the monolithic primary segments has an exclusive secondary conjugate of $1.05 \mathrm{~m}$ in diameter, hanging from $\sim 30 \mathrm{~m}$ high from the primary. Also, the set of secondaries are optically conjugated to $\sim 160 \mathrm{~m}$ high air layer, which enables very high quality ground layer adaptive optics corrections.

The combination of telescope's elements makes the beam reach the second focus as an F/8 beam, a very fast beam-speed compared with the other two ELT's, the TMT and E-ELT, yielding a plate scale of $1 \mathrm{~mm} /{ }^{\prime \prime}$, which, in turn, allows GMT to have wide field-ofview instruments, something that other ELTs are not able to do. Another characteristic of this design, that also facilitates the use of wide-field instruments, is a curved focal plane towards the instruments (concave focal plane). A comparison between the three ELTs is shown in table 1.2 .

Table 1.2 - Comparison of the three ELTs. (private communication, Bernstein)

\begin{tabular}{c||c|c|c} 
& GMT & TMT & E-ELT \\
\hline \hline Aperture & $24.5 m$ & $30 m$ & $39.3 m$ \\
\hline Collecting Area & $368 m^{2}$ & $655 m^{2}$ & $978 m^{2}$ \\
\hline Final Focal & $\mathrm{F} / 8$ & $\mathrm{~F} / 15$ & $\mathrm{~F} / 17.5$ \\
\hline Focal Plane Scale & $1.0 \mathrm{~mm} /{ }^{\prime \prime}$ & $2.2 \mathrm{~mm} /{ }^{\prime \prime}$ & $3.6 \mathrm{~mm} /{ }^{\prime \prime}$ \\
\hline Field of View & $\begin{array}{c}10^{\prime} \\
\left(20^{\prime} \mathrm{w} / \mathrm{cor}\right)\end{array}$ & $\begin{array}{c}10^{\prime} \\
\left(15^{\prime} \mathrm{w} / \mathrm{cor}\right)\end{array}$ & $\begin{array}{c}7^{\prime} \\
\left(10^{\prime} \mathrm{w} / \mathrm{cor}\right)\end{array}$
\end{tabular}

\subsubsection{G-CLEF Overview}

The first light instrument of GMT is the GMT - Consortium Large Earth Finder (GCLEF). It is an optical high resolution echelle spectrograph; it will be able to observe wavelengths ranging from 3500 to $9000 \AA$, with resolutions from 20.000 to 110.000 , depending on the observing mode. Because the first set of M2 will be delivered without Adaptive Optics (section 1.3) capability, G-CLEF is designed to be a seeing-limited instrument.

Since G-CLEF will be the one of first instrument on GMT, its design is intended to address a broad range of the most urgent astrophysical problems, taken as priorities By the GMT scientific community, such as census and characterization of the most metal poor stars, evolution of and abundances of galaxies and probing the intergalactic and interstellar medium at high redshifts (Szentgyorgyi et al., 2012). However, the instrument was idealized 
with a very clear main goal in mind: find and characterize Earth-sized exoplanets orbiting Solar-like stars.

To reach the stability required so it can deliver the desired precision, it needs to sit in a gravity invariant, vacuum-enclosed and temperature controlled environment; it will, therefore, reside in the azimuth platform of the telescope, as illustrated in figure 1.3, and be fiber-fed.
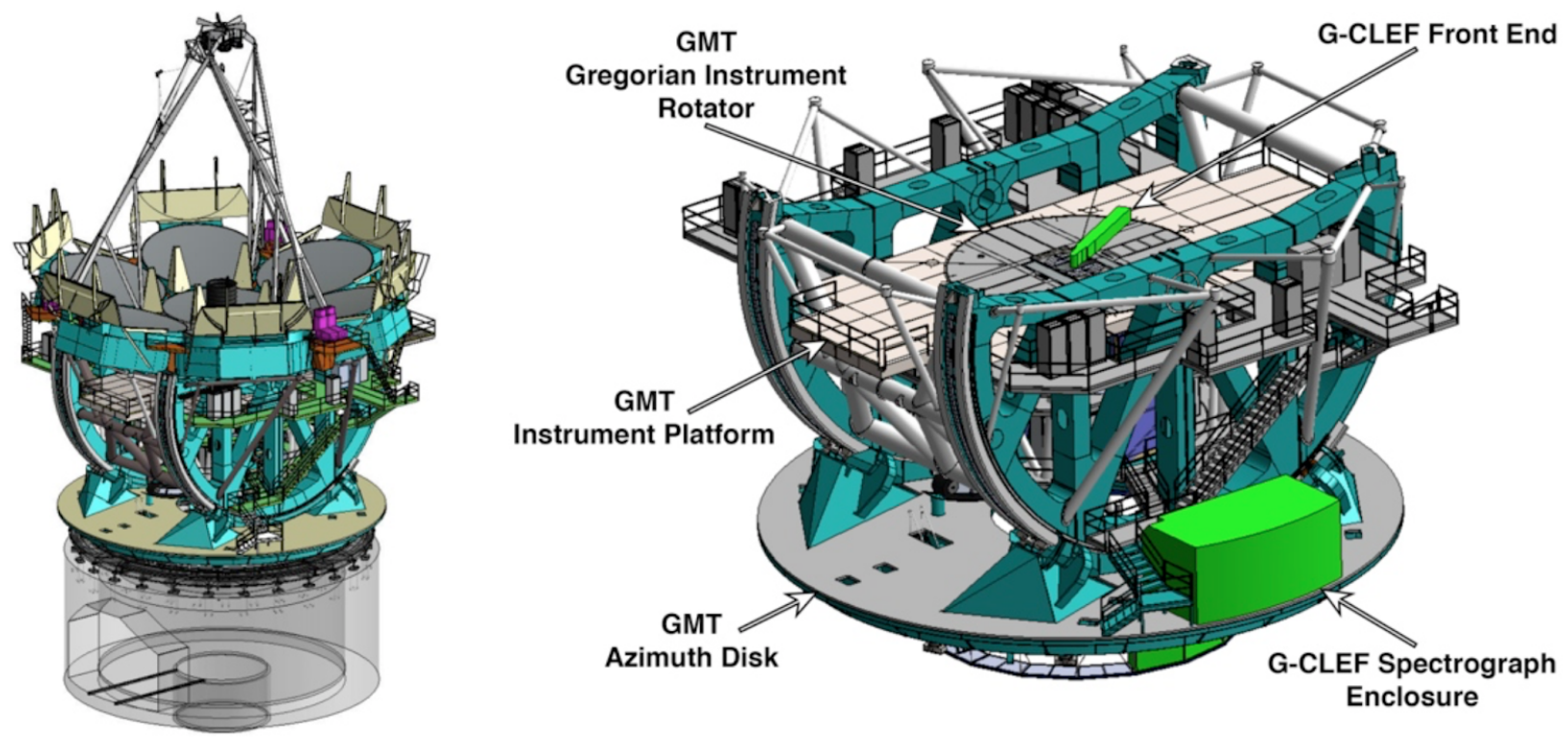

Figure 1.3: G-CLEF residing in the Azimuth Platform of GMT. Connection between instrument and telescope focus is done through optical fibers. Figure 1 from Szentgyorgyi et al. (2014).

Nowadays Kepler's sample dominates the catalogue of transiting exoplanet candidates with faint objects, which are not suitable for mass determination of low mass exoplanets through precision radial velocity measurements nor its atmospheres studies. However, prior to the start of GMT's activities, the Transiting Exoplanet Survey Satellite (TESS) and CHaracterising ExOPlanet Satellite (CHEOPS) will be launched and will provide a large sample of bright stars being orbited by low-mass transiting planets, which are great targets for G-CLEF. It is true that some current and upcoming instruments on $3.5 \mathrm{~m}-10 \mathrm{~m}$ telescopes like ESPRESSO, CARMENES, EXPRES and Maroon-X will have access to TESS and CHEOPS catalogue before G-CLEF, nevertheless, many discoveries will only be possible with GMT's aperture, for example finding traces of $\mathrm{O}_{2}$ biomarkers in these exoplanets' atmospheres (Rodler and López-Morales, 2014; Szentgyorgyi et al., 2016). 


\subsubsection{Observation Modes}

G-CLEF will run several Observing Modes, each of which with a specific fiber diameter and, hence, resolution. Each observing mode will address different cases of study; some examples of it, together with the parameters of each mode are displayed in table 1.3.

The Precision Radial Velocity (PRV) mode will offer the best precision the instrument is able to obtain; such resolution is capable of resolving features of F, G and K stars. To reach such a precision with this resolution the instrument needs to use a double scrambling system; how this system works is explained in section 1.4.2. Other research interests, such as high redshift cosmology does not require the scrambling functionality, but can still benefit from PRV resolution, therefore there is a similar mode, the Non-Scrambled PRV (NS-PRV) mode that provides the same resolution but with higher throughput.

Still, not all cases of study require such high resolution, and for those cases there are observation modes that have a compromise between resolution and throughput. Since their required resolution is lower than that achievable by the instrument, the slit can be broadened up (use bigger fiber) to increase throughput in detriment of resolution; those modes are: High-Throughput (HT) mode, with low-resolution, and Medium-Resolution (MR) mode, with lower throughput when compared with HT.

G-CLEF will also support Multi-Object (MOS) observation with MANIFEST (ManyInstrument Fiber System). Each exposure of this mode will be able to capture full spectral coverage of $\sim 5$ objects or partial coverage of up to $\sim 40$ objects.

\subsubsection{Optical System - Spectrograph, Cameras and Detectors}

The spectrograph design is an asymmetric, two-channels, white pupil design. As we can see on figure 1.4, the instrument is fed through the fiber slit, the beam gets collimated by an off-axis parabola mirror (M1), dispersed the first time by the echelle grating, then refocused by M1 again, redirected by a cylindrical margin fold mirror and re-collimated by the pupil transfer mirror (M2).

The echelle grating is a $3 \mathrm{X} 1$ mosaic of $400 \mathrm{~mm} \times 300 \mathrm{~mm}$ gratings, with ruling density of 31.6 line $/ \mathrm{mm}$, working in Quasi-Littrow configuration. The Margin mirror also works as a field flattener for the system. M2 function is to re-collimate the beam, but also it is used to decrease the focal length of the system, from $2400 \mathrm{~mm}$ (M1) to $1600 \mathrm{~mm}$, therefore, 
Table 1.3 - G-CLEF's observation modes and its parameters. Throughput requirements at 650nm, as described in Podgorski (2018) throughput error budget report.

\begin{tabular}{c|c|c|c|c|c} 
Mode & Resolution & $\begin{array}{c}\text { Throughput } \\
\text { Requirement }\end{array}$ & Fiber & $\begin{array}{c}\text { \#Science } \\
\text { Fibers }\end{array}$ & Case of Interest \\
\hline \hline PRV & 108.000 & $5.9 \%$ & $\begin{array}{c}\text { Octagonal } \\
100 \mu m\end{array}$ & 7 & $\begin{array}{c}\text { Low-mass exoplanets } \\
\text { Atmosphere studies }\end{array}$ \\
\hline NS-PRV & 108.000 & $7.3 \%$ & $\begin{array}{c}\text { Circular } \\
100 \mu m\end{array}$ & 7 & High-Z Cosmology \\
\hline MR & 35.000 & $7.3 \%$ & $\begin{array}{c}\text { Circular } \\
300 \mu m\end{array}$ & 1 & Stellar abundance \\
\hline HT & 19.000 & $11.5 \%$ & $\begin{array}{c}\text { Circular } \\
450 \mu m\end{array}$ & 1 & High redshift quasars \\
\hline MOS & 35.000 & $7.3 \%$ & $\begin{array}{c}\text { Circular } \\
300 \mu m\end{array}$ & Up to & Local Group Archaeology \\
& & & & &
\end{tabular}

also decreasing the pupil size to a factor of $2 / 3$, allowing the camera systems to be smaller and, consequently, cheaper; the beam hits the echelle grating with a diameter of $300 \mathrm{~mm}$ and, after M2, it can enter the camera systems as a $200 \mathrm{~mm}$ beam.

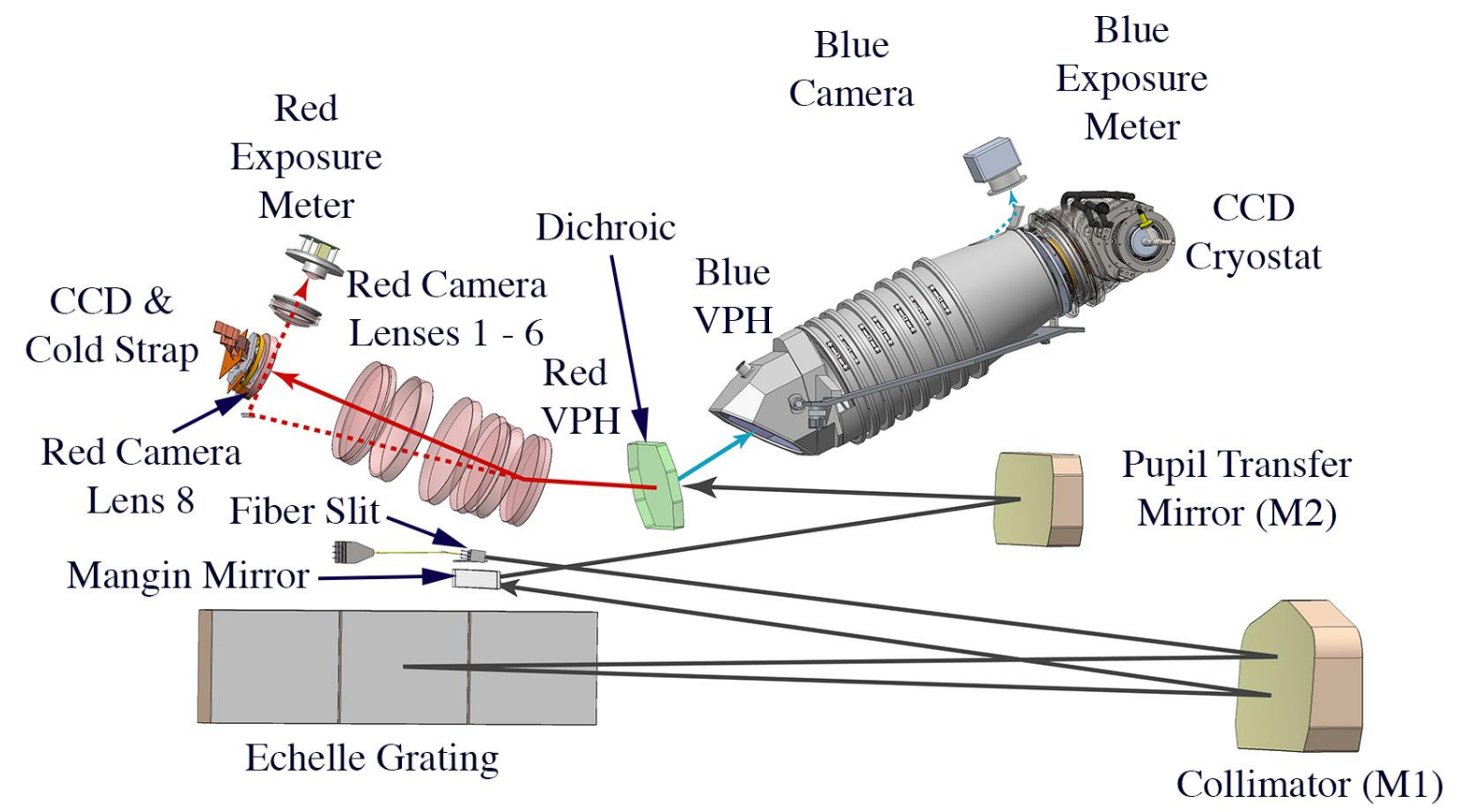

Figure 1.4: Spectrograph optical design. Dashed line shows the zero order (light that was not deviated by the VPH). Solid line shows light path throughout the spectrograph system. Figure 4 from Szentgyorgyi et al. (2016). 
The camera's subsystem is divided into 2 channels, a blue and a red one, splitting the beam at $5400 \AA$ by a dichroic, in the parallel beam.

The greater the number of camera arms, the more complex and more expensive the system becomes. However, when the system is divided into more than one channel, it becomes easier to design more efficient and optimized systems for each wavelength range, allowing it to preserve a good response on both, blue and red, ends of the spectrum.

The cross dispersers are Volume Phase Holographic (VPH) gratings, positioned right after the dichroic, in each channel, before entering the camera lens system. The blue channel VPH is a 910 line/mm-grating, while the red one is a 370 line $/ \mathrm{mm}$.

Both detectors, each of them optimized for its channel wavelength range, is a $10 \mathrm{k} \mathrm{x}$ 10k CCD, and each has pixel size of $9 \mu m$ x $9 \mu m$ (STA 1600).

\subsubsection{Fiber-feed System for Precision Radial Velocity Mode}

The precision radial velocity mode has its own system to feed the fibers. It needs it because this mode works with a pupil slicer. The pupil of the telescope will be divided into seven apertures, each of which corresponding to a GMT primary mirror segment. After slicing the beam, which comes as an $F / 8$ from the telescope, each new section of the beam is turned into an $F / 3$, that then feeds an individual $100 \mu m$ round fiber. The pupil slice is done so that when the fibers get to G-CLEF, they can be reorganized to form a pseudo-slit, with width of $100 \mu m$, as seen in figure 1.6. Reducing the beam speed of the beam feeding the fiber is done in order to minimize focal ratio degradation light losses. This will be addressed in section 1.4.1. It can be seen in figure 1.5. 

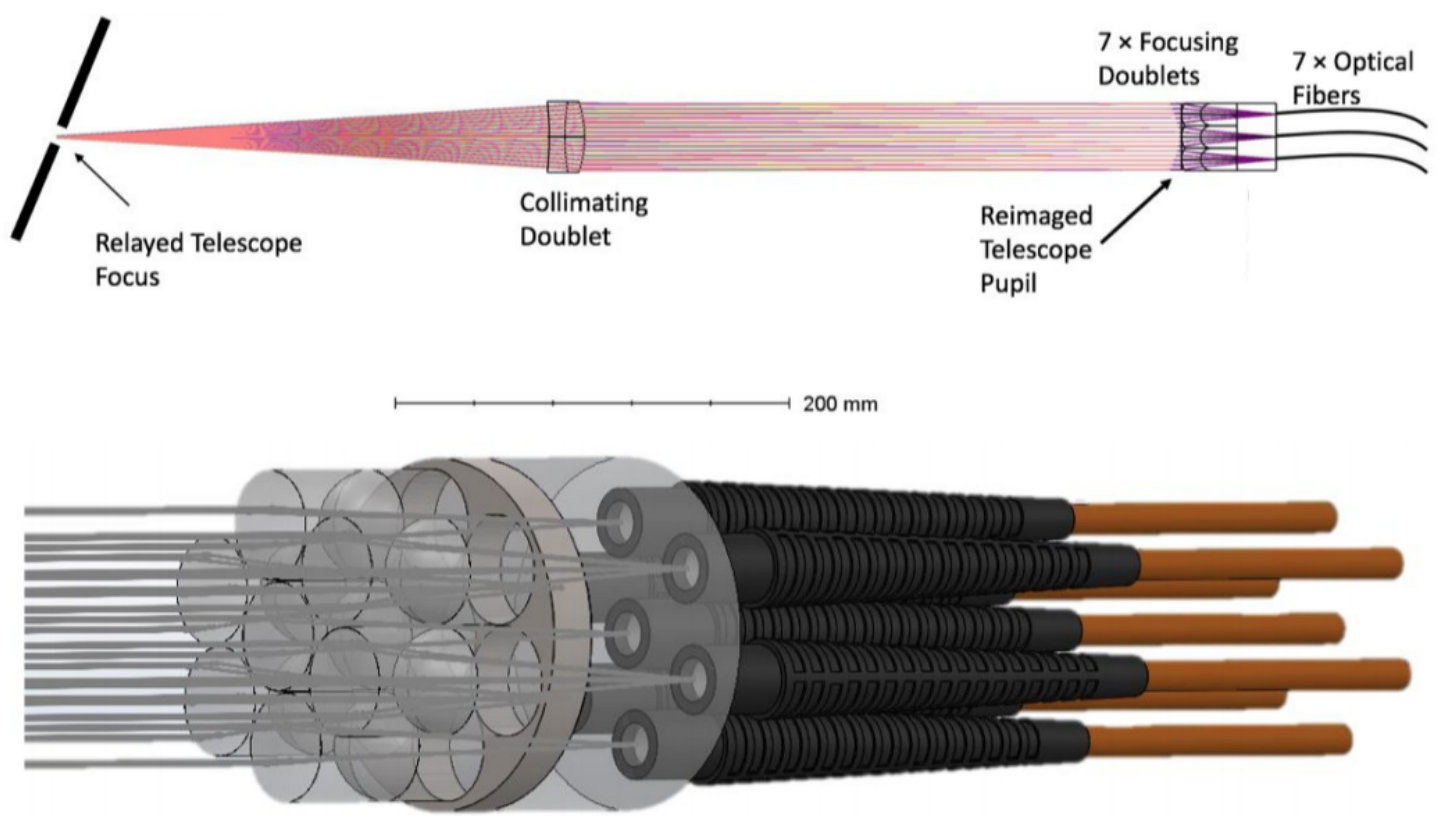

Figure 1.5: Fiber-fed system: each GMT mirror segment will feed a single $100 \mu \mathrm{m}$ fiber on PRV and NS-PRV mode. Figure 5 from Szentgyorgyi et al. (2018).

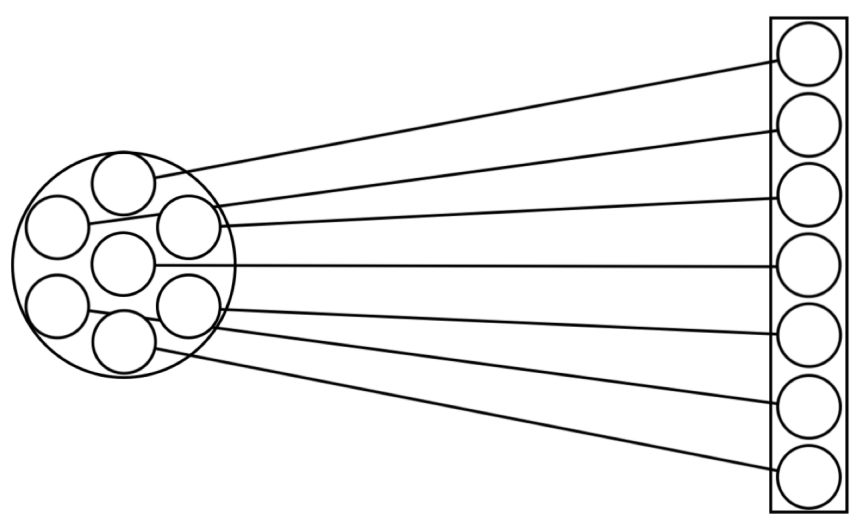

Figure 1.6: Pupil slice allows to reorganize the fibers, from the shape of GMT's pupil $(\sim 300 \mu m$ in diameter) to a slit with width of $100 \mu m$.

\subsection{Adaptive Optics}

All astronomical observations, in the optical and infra-red, when conducted from a ground-based telescope, suffer from atmosphere interference. That interference happens in more than one way: (1) thermal and line emission, which creates a background noise, usually called sky brightness, (2) refraction, which disperses the image spectrally and shifts its position, (3) extinction, which dims down the flux of the object, and (4) turbulence, 
that blurs the image, also called seeing (Bely, 2003).

Seeing is a result of the movement of atmosphere layers caused either by wind or convection, which makes the atmosphere layers move. These layers often have different temperatures, which also lead to different indices of refraction. These slight differences in the refraction index and the layers' movement causes the seeing.

Since astronomical targets are so far away from Earth we consider their light to get to us well collimated and with flat (in phase) and parallel wavefronts. Wavefronts are defined as perpendicular planes, distanced one from the other by $\lambda$ (where $\lambda$ is the observed wavelength), moving in the same direction as the radiation. When it hits the atmosphere, the differences in indexes of refraction make light go through different optical paths and that causes the wavefront to be distorted, as shown in figure 1.7.

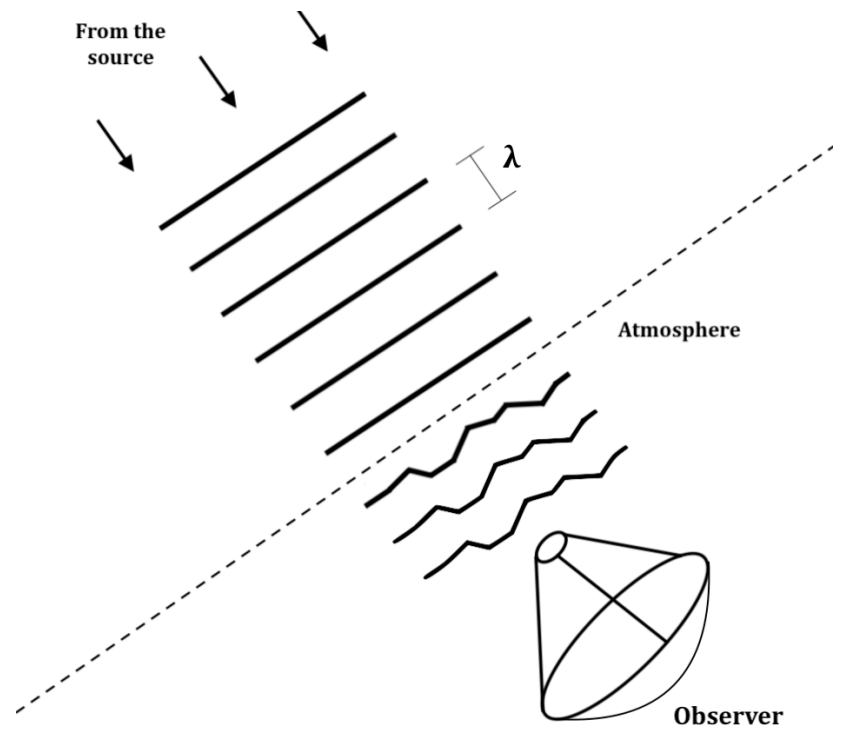

Figure 1.7: Wavefront being disrupted by atmospheric turbulence.

A simple way to characterize turbulence strength is the Fried parameter, also called Fried's coherence length, $r_{o}$. It is a statistical parameter defined as the aperture size over which the wavefront is able to pass without being disrupted, which means that within this aperture the wavefronts are kept in phase and parallel to each other.

The seeing increases as $r_{o}$ decreases. It is easy to picture it in an exaggerated example such as $r_{o} \rightarrow \infty$, where the 'whole' wavefront would have space to go by undisturbed. As opposed to $r_{o} \rightarrow 0$, where the wavefront would be disrupted 'infinite' times through its extension. The seeing, in good observatory sites, usually varies from $\sim 0.6^{\prime \prime}$ to $\sim 1.0^{\prime \prime}$, and $r_{o}$ from $20 \mathrm{~cm}$ to $10 \mathrm{~cm}$ (at optical wavelengths), respectively. Since $r_{o}$ vary directly 


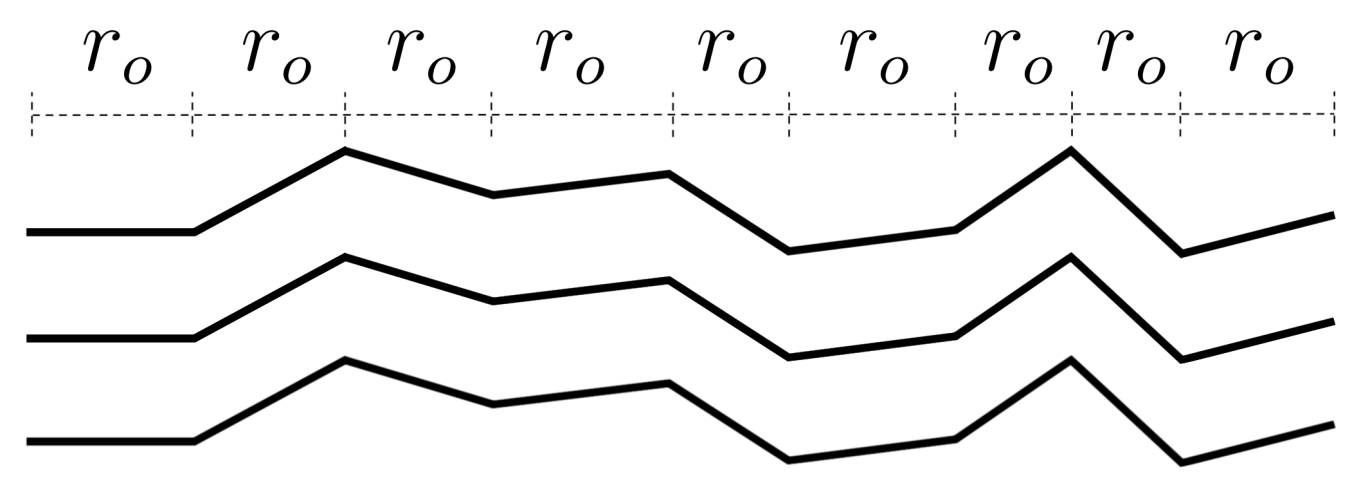

Figure 1.8: Oversimplified scheme of disrupted wavefront and Fried's coherence length, $r_{o}$.

with the indices of refraction of the atmosphere's layers, it is natural to say that it will be wavelenght dependent, as show in equation 1.1 from Fried (1966).

$$
r_{o} \propto \lambda^{\frac{6}{5}}
$$

The angular resolution of an optical system is bound by the aperture of the system and the wavelenght in which the observation is conducted, as shown in equation 1.2.

$$
\theta=1.22 \frac{\lambda}{D}
$$

That means that a system with aperture $D$ in diameter, when observing in wavelenght $\lambda$ is able to differentiate two objects that are $\theta$ radians distant from each other, if they are closer than that, the system sees it as only one object. This relation is only valid for diffraction-limited systems, such as space telescopes (or telescopes with $D<r_{o}$ ). Ground based telescope's resolution is limited by the seeing and therefore its resolution becomes, roughly:

$$
\theta=1.22 \frac{\lambda}{r_{o}}
$$


There is one main way to face the seeing problem in order to aim for a diffraction-limited performance, other than having a space telescope: using adaptive optics corrections. Adaptive Optics (AO) is the system responsible fot measuring the atmospheric disturbances on the beam and correcting it, in a high-frequency regime. This is done while doing the observation.

The beam gets into the optical system where it is split, most of the light goes to the science instrument, where it is studied, and the other portion goes to the wavefront sensor (WFS), which is responsible for measuring how disrupted the wavefront is on that moment. The sensor, then, processes the information and the control system applies the opposite distortion on the deformable mirror, in order to try to compensate whatever disruption was caused by atmospheric turbulence on the beam, as seen in figure 1.9. Since the atmosphere is dynamic this process needs to be done in an iterative way and with high frequency, on the order of $1,000 \mathrm{~Hz}$.

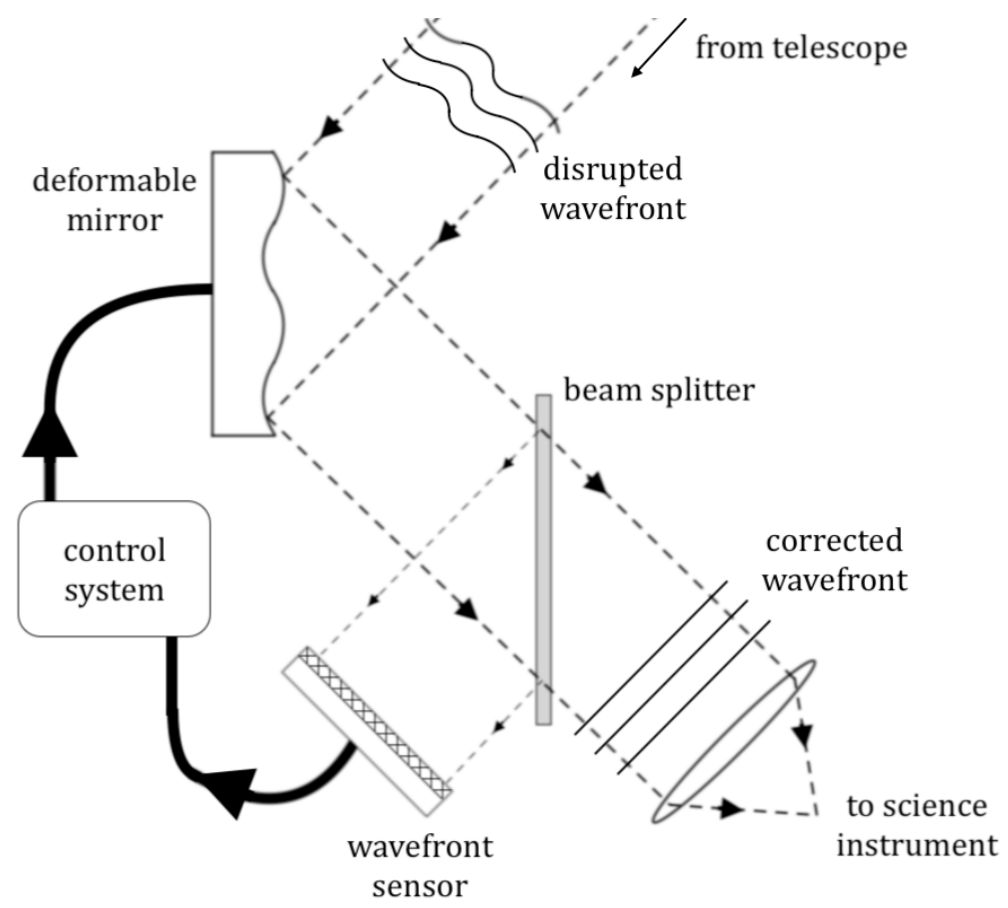

Figure 1.9: Schematics of adaptive optics system.

There are several methods of measuring and corrections, and each of them is able to correct different levels of disturbances. A few examples are listed bellow:

Tip-tilt : Lowest level of AO correction. It does not aim to enhance the point-spread- 
function (PSF) of the observation, but rather only correct the movement caused by the atmosphere in the image.

GLAO : Ground Layer Adaptive Optics is a low-order correction that aims to correct the layers closer to the ground, which is responsible for great part of the turbulence, around $60 \%$, (Hubin et al., 2005).

High-order : Responsible for probing higher layers of the atmosphere and correcting higher-order errors.

In order to probe the information from the atmosphere, the system needs bright sources. At times, bright stars end up inside the observed field-of-view. If that happens that star can be used as guide for the AO system (Natural Guide Star; NGS). If that is not the case, a Laser Guide Star (LGS) has to be implanted in the atmosphere in order for the system to have a proper target to observe. For example, one way to do that is using sodium lasers that excite sodium atoms of the mesosphere, creating an artificial star as pictured in figure 1.10 .

The performance of an AO system can be quantified by its Strehl ratio. It is the ratio between the maximum of intensity of the PSF with the adaptive optics system working and the PSF in a diffraction limited case, as imaged in figure 1.11. The example exposed in figure 1.11 was measured in the optical lab, and the Strehl ratio measure for the AO system simulated was 0.25 . 


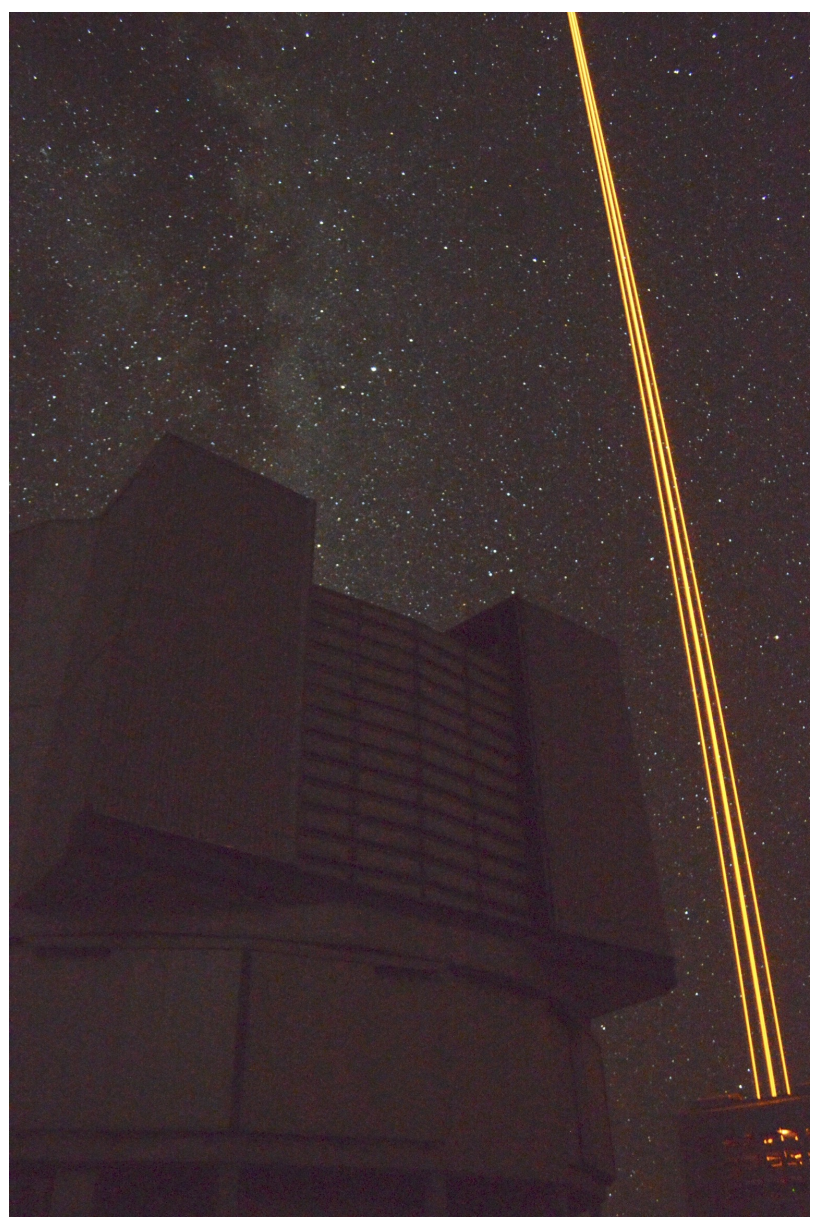

Figure 1.10: Very Large Telescope's (VLT) UT4 using sodium lasers to enable AO correction.

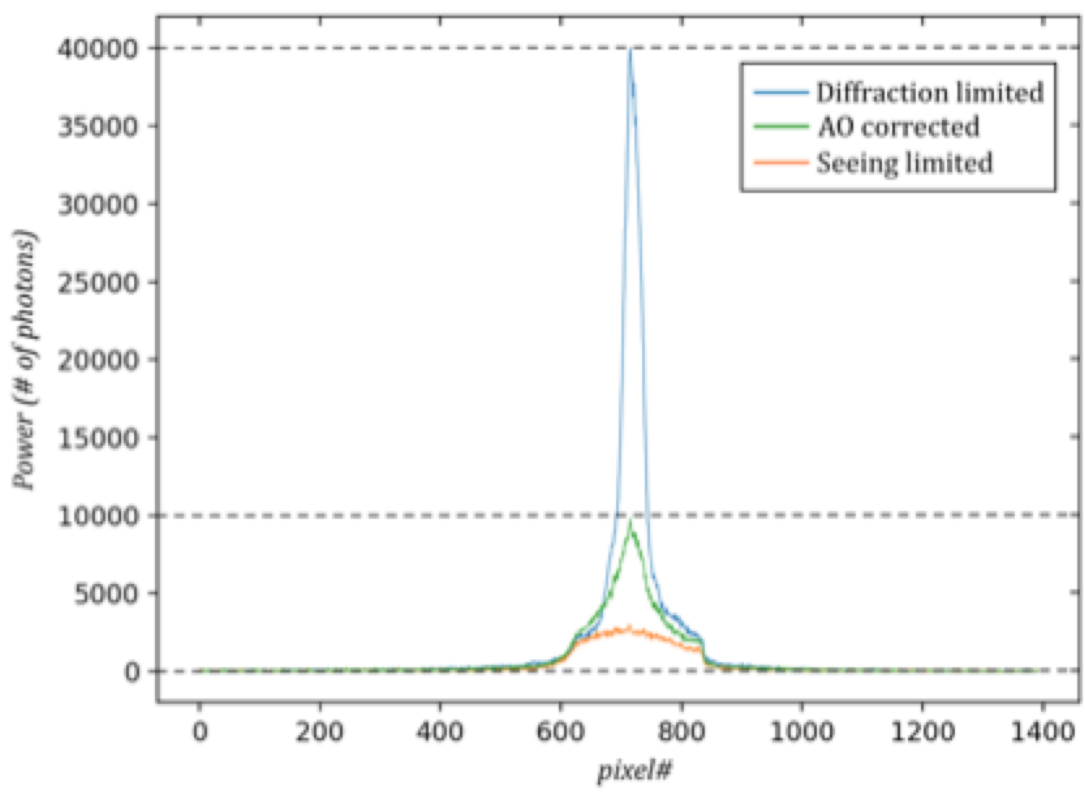

Figure 1.11: Point-spread-function of three scenarios: diffraction limited case, AO corrected and seeing limited. 


\subsubsection{Lexitec Phase Screens}

Throughout this thesis, when describing the experiments we performed, we will be citing phase screens. These phase screens are manufactured by Lexitek Inc.. They are acrylic plates sandwiching an optical polymer, which is responsible for causing a disruption of the optical beam. This optical polymer is composed by two layers of materials of similar, but unequal, indices of refraction $\left(n_{1}\right.$ and $\left.n_{2}\right)$, with the interface between them following a profile $h(x)$ (specified prior to manufacturing), as seen in figure 1.12.

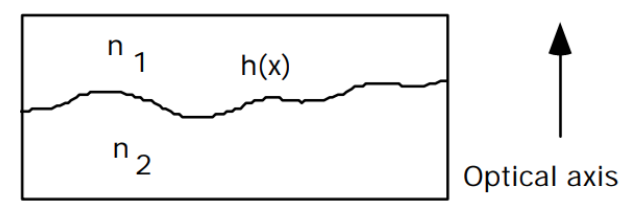

Figure 1.12: Phase screen: interface between polymers of slightly different indices of refraction. Figure from Lexitek Inc..

This profile will cause differences among the optical paths throughout the plate, and therefore, the light passing through it will have its wave front disrupted; one can make the beam be disrupted in controlled conditions choosing $h(x)$ in the design.

In the experiments, two phase screens are used, provided by Harvard-Smithsonian Center for Astrophysics (CfA): one that mimics atmospheric turbulence (figure 1.13 left panel) and another that contains a simulation of atmospheric turbulence after being corrected by adaptive optics (figure 1.13 right panel). On the AO case there are two different simulations on the phase screen: inner circle has corrections by a Natural Guide Star AO (NGSAO) system (high order correction), and, on the outer annular region, by a Ground Layer AO (GLAO) system (low order correction).

The turbulence imprinted on the uncorrected phase screen has a Fried's parameter of $r_{o}=0.39 \mathrm{~mm}$. Therefore, in order to simulate the median seeing on Las Campanas site (home of Magellan Telescope and future home to GMT) of 0.6 " (or $r_{o} \sim 17 \mathrm{~cm}$ ) hitting one of the $8.4 \mathrm{~m}$ segment of GMT mirror we need to define the size of the entrance pupil (I1 in figure 4.1 ) as $20 \mathrm{~mm}$. In addition, in order for it to act in the same way as atmospheric turbulence does, it has to be moving and not just be a static disruption; we mimic so by rotating it. We use a motor that holds the phase screen and spins, with the desired speed. It is also possible to set it to do a chosen number of full spins. 


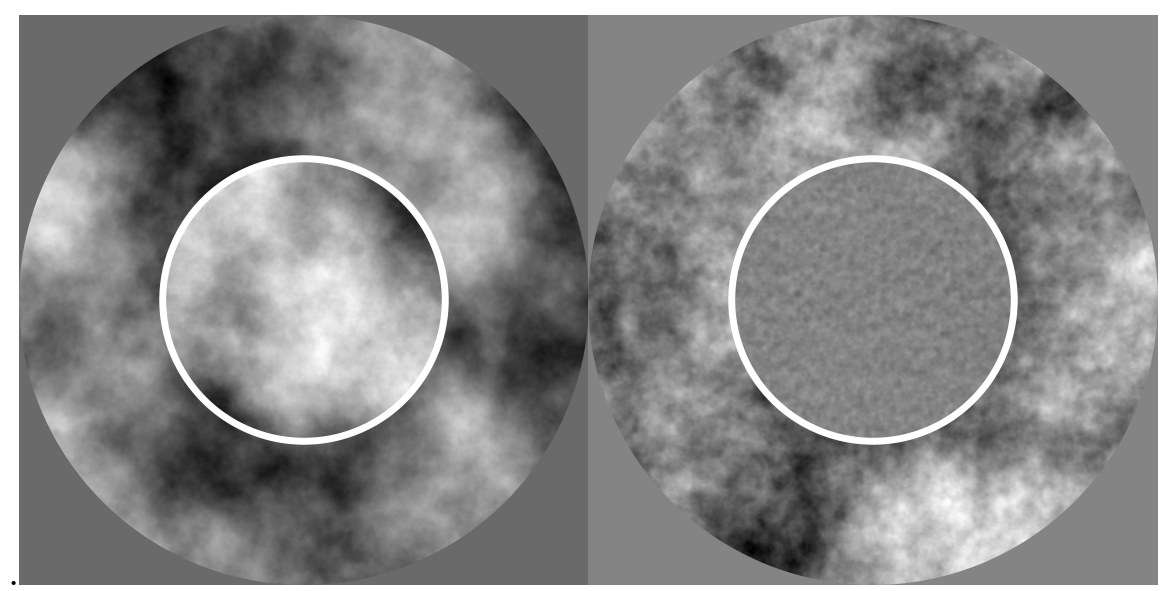

Figure 1.13: Left: Seeing phase screen; Right: AO corrected phase screen.

\subsection{Optical Fibers}

Optical fibers have been used in astronomical observations, propagating the light coming from the telescope to an instrument, very successfully, since the late 1970s . Fibers can be used is several different observing modes, such as precision radial velocity, multiobject spectroscopy, integral-field units (IFU), interferometry and photometry ((Parry, 1998)).

Usually, fibers can be understood as glass tubes that propagate light; it can be divided into three structures: core, cladding and buffer, pictured in figure 1.14.

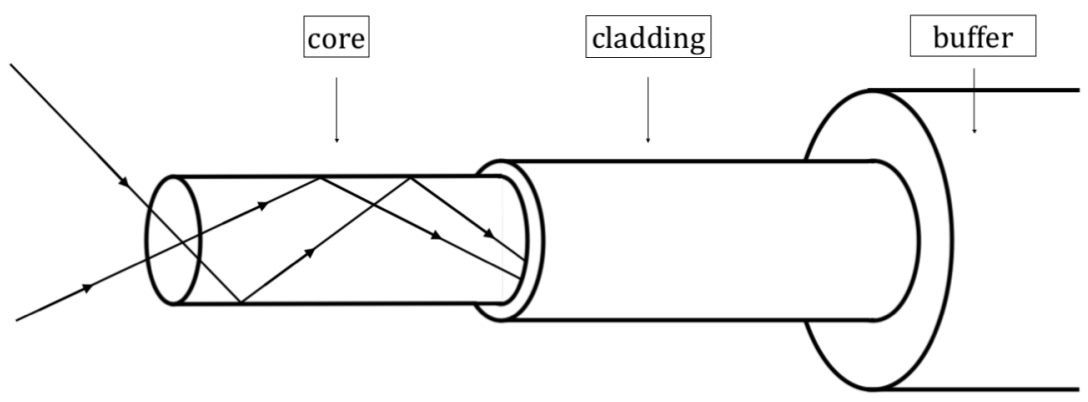

Figure 1.14: Optical fiber structure.

A simple way to understand how optical fibers work is imagining light rays traveling through the core, bouncing off the cladding's surface at small angles with respect to the fiber's optical axis. The light gets reflected at the interface between core and cladding 
because the cladding's index of refraction is lower than that of the core, not allowing any light to escape. While the buffer offers mechanical protection for the inner structure.

The main characteristics that make optical fibers so useful in astronomy are: flexibility, high scrambling capability, ability to spatially reorganize the input into the instrument and its ability to enable that telescope and instrument focal planes are in different positions. The latter permits G-CLEF, for example, to sit on the azimuth platform of GMT enabling an extremely stable environment for the spectrograph. The ability to reorganize the input of light into the instrument is extremely useful for multi-object spectroscopy, since, without it, some objects' spectra may end up overlapping, once the sources are randomly scattered on the field of view; also for IFU, since the goal is to take spectra of $2 \mathrm{D}$ fields (or extended sources), it would not be possible without, somehow, slicing the image into slits.

\subsubsection{Effects on Beam}

Optical fibers are decent scramblers, which means that the light loses almost all its spacial information when propagating through it, yielding a fairly homogeneous light distribution coming out of the fiber, which tends to be great for spectrographs. However, in extreme cases, such as G-CLEF's, the scrambling provided by the fiber is not enough to deliver the required beam stability and precision. As said in section 1.2, G-CLEF is designed to be a seeing limited instrument; because of that, the light feeding the fiber will be disrupted, over time, by the atmosphere, and the instrument, as sensitive as it is, would be exposed to these variations on the detector, limiting its measurements precision. Fortunately there is a method called Double Scrambler that, as the name suggests, scrambles the light even further, in order to desensitize even more the beam to the input profile instability (section 1.4.2). Octagonal fibers are better scramblers than circular ones hence the use of them for PRV mode (Avila, 2012). Its use is still relatively new in astronomy, and because of that there is still not a consensus on what are the downsides of its use.

When the beam goes through an optical fiber it suffers Focal Ratio Degradation (FRD), which changes the beam speed or $F /$ \# of the beam to a faster one, as seen on figure 1.15, as a result of stress, micro-bend and irregularities at the fiber surface (Barden, 1998; Belland et al., 2019). The problem with this is that it does not preserves the $A \Omega$ product (etendue), it is increased. Which means that, although the total energy of the system is mostly preserved, the light density is not. This means that the information is also more 
spread in space, which, in turn, translates into instrument loss of performance. One way to minimize this issue is to feed the fiber at low $F / \#$, once FRD is less of a problem for slower beam speeds (Barden, 1995).

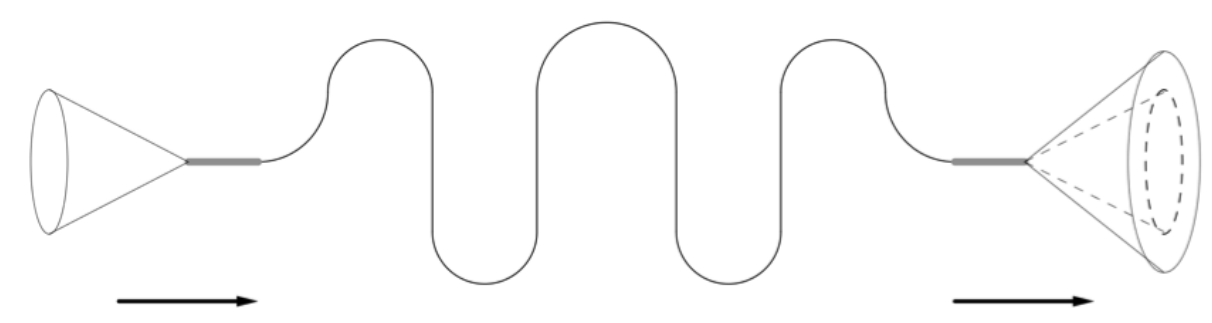

Figure 1.15: Optical fiber FDR scheme.

Another issue that fiber users face is that fibers transmits differently for different wavelengths. However, that is easily solved by calibrating the fiber. Related to that, there is also internal absorption losses. Fibers are, usually, strong absorbents in the blue part of the spectrum. Since G-CLEF will reside in the azimuth platform, the fiber cable that connects instrument and telescope will only have $17 \mathrm{~m}$, minimizing absorption of the blue light and enabling G-CLEF's passband to reach wavelengths as short as $3500 \AA$, due to decreased internal transmission of the optical fiber.

\subsubsection{Double Scrambler}

The Double Scrambler (DS) is a simple device that consists of a single optical element, a ball-lens and it has the purpose of switching the near and far field of the fiber.

Near-field is the light intensity distribution on the fiber face (focal plane), while far-field is the angular distribution on the pupil plane.

The idea is to use two fibers, with the DS in between them, so the DS would feed the second fiber with the first fiber far-field, basically exchanging angle and position as imaged in figure 1.16, losing spatial information, and consequently yielding a more homogeneous and desensitized beam as the output of the second fiber. A beam is considered desensitized when a shift in the centroid of the beam feeding the fiber translates, on the beam coming out of the fiber, to a smaller centroid shift. For example, if the centroid of an input beam shifts by $10 \mu m$ and the output centroid is shifted by $0.01 \mu m$, this beam is desensitized by a factor of 1,000 . 


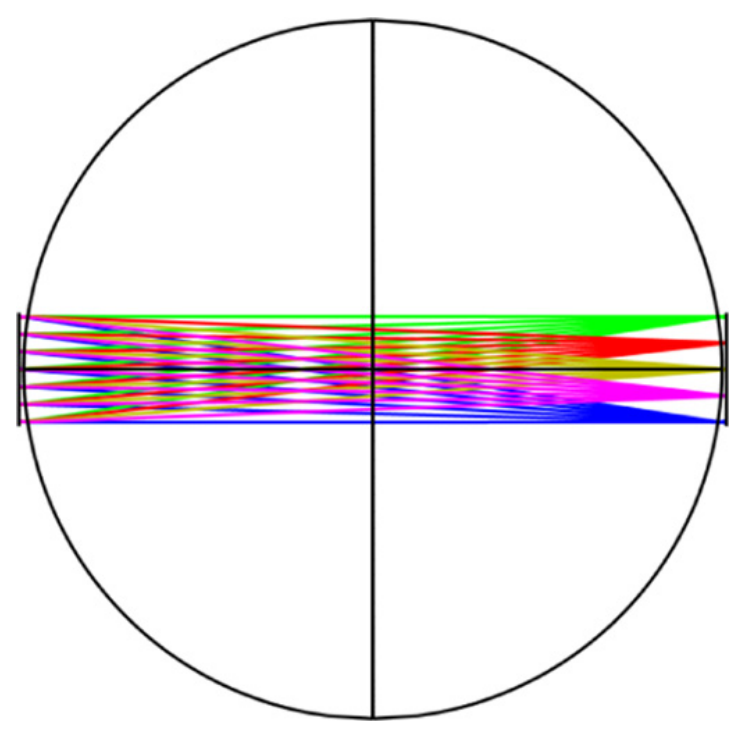

Figure 1.16: Ray traces through double scrambler lens. Figure 12 of Halverson et al. (2015).

The reason why the DS is so important for precision radial velocity measurements is exactly the desensitization of the beam that it allows, which is crucial for doing the precise measurements needed to detect changes in the movement of stars over a long period of time, given that the light that reaches the instrument has to be ideally independent of any changes introduced by atmosphere, guiding and tracking errors. This is, however, not as important for measurements in cosmology studies. As written in section 1.2, this specific science goal can take advantage of PRV mode resolution but it does not need the DS functionality, since the phenomena studied in the field are not time variable and therefore are not as sensible to variations in the atmosphere as the measurements of stellar precision radial velocity. G-CLEF's DS device is supposed to desensitize the beam by a factor of 3,500 .

This chapter covers the basic concepts that are needed to fully comprehend the work done in the context of this thesis. Chapter 2 shows the motivation we had for this work. Chapter 3 presents a work done in collaboration with Dr. Mello. Chapter 4 describes how the experiments for this thesis were designed, assembled and also how the measurements were taken, chapter 5 shows the analyses and results for each experiment and finally chapter 6 presents our conclusions. 


\section{Motivation}

G-CLEF is designed to be a seeing-limited instrument, which means that its desired performance can be achieved without the use of an AO system. This constrain was set upon the project due to the fact that G-CLEF is expected to be a first-light instrument and, during commissioning, GMT will not have adaptive secondary mirrors, which makes it unable to perform adaptive optics corrections. However, it is intuitive to expect that, with $\mathrm{AO}$ correction, the instrument performance would improve. In fact, at an early stage of the project, part of the G-CLEF's team - Marcos Van Dam, Brian McLeod and Gabor Furesz - made an effort to study and evaluate the benefits of adaptive optics for the instrument. This is described in the document "Evaluating the benefits of adaptive optics for G-CLEF: snapshot of the study", hereafter, early study.

G-CLEF's front end configuration for the PRV mode offers an easy way of operating a near-infrared (NIR) wavefront sensor without compromising any of the signal (a sensor in the optical band would also be possible by compromising $\sim 5 \%$ of the signal, and it would yield a higher gain). In fact, it would take no modification in the optical design apart from the insertion of a dichroic beam splitter and the WFS itself.

This early study was a simulated-based study: they simulated G-CLEF working without $\mathrm{AO}$ and compared it with the simulation working with several different methods of correction (tip-tilt sensor, GLAO and High-order sensor). They found the parameters shown in tables 2.1, 2.2 and 2.3 and illustrated in figures 2.1 and 2.2.

The exact information of what details were taken into consideration for these simulations are not present in the manuscript, therefore it was not possible to reproduce it exactly in the lab. Still, the most important information are presented, which is the throughput and variability of the beam as a function of wavelength, correction method and 


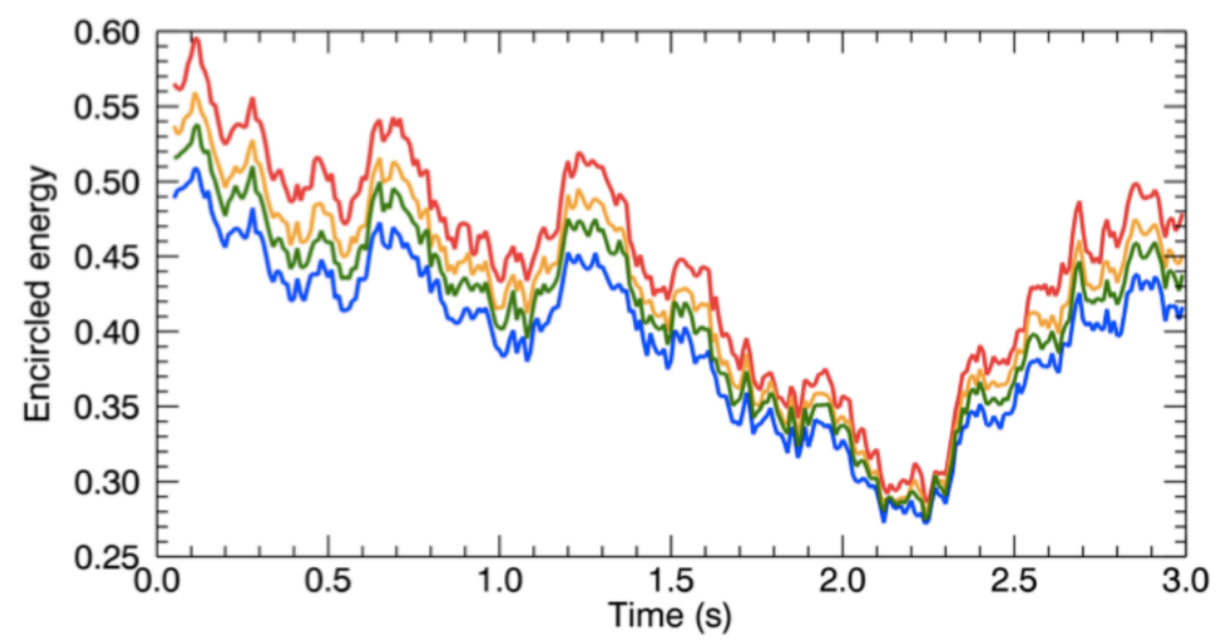

Figure 2.1: Variation in encircled energy over time for an uncorrected wavefront as a function of wavelength: $440 \mu \mathrm{m}$ (blue), $550 \mu \mathrm{m}$ (green), $640 \mu \mathrm{m}$ (yellow) and $790 \mu \mathrm{m}$ (red).

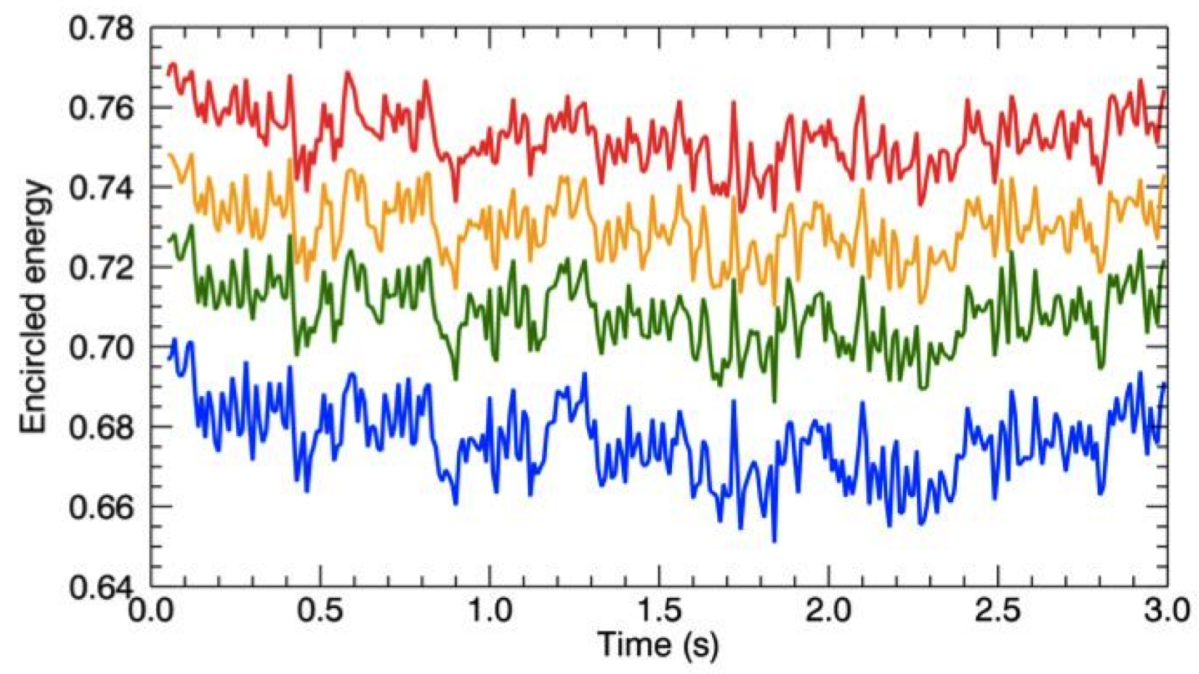

Figure 2.2: Variation in encircled energy over time for an $\mathrm{AO}$ corrected wavefront as a function of wavelength: $440 \mu \mathrm{m}$ (blue), $550 \mu \mathrm{m}$ (green), $640 \mu \mathrm{m}$ (yellow) and $790 \mu \mathrm{m}$ (red).

Table 2.1 - 700 mas encircled energy as a function of wavelength and wavefront correction strategy.

\begin{tabular}{l||c|c|c|c} 
Wavelegth $(\mu m)$ & 440 & 550 & 640 & 790 \\
\hline No sensor & 0.3949 & 0.4126 & 0.4254 & 0.4458 \\
Tip-tilt sensor & 0.4935 & 0.5215 & 0.5417 & 0.5723 \\
GLAO & 0.5163 & 0.5461 & 0.5678 & 0.6000 \\
High-order sensor & 0.6764 & 0.7086 & 0.7302 & 0.7527
\end{tabular}

seeing strength. This could be then used as base for comparison with the optical bench experimental results.

The early study found an increase in throughput of almost a factor of 2 , when using 
Table 2.2 - Variability in 700 mas encircled energy as a function of wavelength and wavefront correction strategy.

\begin{tabular}{l||c|c|c|c} 
Wavelegth $(\mu m)$ & 440 & 550 & 640 & 790 \\
\hline No sensor & 0.0561 & 0.0617 & 0.0660 & 0.0725 \\
Tip-tilt sensor & 0.0229 & 0.0251 & 0.0266 & 0.0287 \\
GLAO & 0.0281 & 0.0308 & 0.0327 & 0.0352 \\
High-order sensor & 0.0095 & 0.0085 & 0.0077 & 0.0074
\end{tabular}

Table 2.3 - 700 mas encircled energy as a function of seeing and wavefront correction strategy. The wave length is $550 \mu \mathrm{m}$

\begin{tabular}{l||c|c|c|c}
$r_{o}$ at $550 \mu \mathrm{m}(\mathrm{m})$ & 0.075 & 0.100 & 0.151 & 0.200 \\
\hline No sensor & 0.1367 & 0.2206 & 0.4126 & 0.5860 \\
Tip-tilt sensor & 0.1800 & 0.2906 & 0.5215 & 0.6922 \\
GLAO & 0.1917 & 0.3093 & 0.5461 & 0.7126 \\
High-order sensor & 0.3277 & 0.4927 & 0.7086 & 0.8117
\end{tabular}

$\mathrm{AO}$ as compared with no AO. Also, they concluded that the use of AO in the early days of GMT's operations can yield a large amount of phasing data for the telescope, enabling the creation of look-up tables to aid later operations. This is feasible due to the fact that G-CLEF's targets are usually bright, which allows them to be also the on-axis guide for the AO system.

After these simulations, the next step that the team had planned was to test the same conditions on an optical bench to confirm what the simulation results had suggested. This thesis is the extension of that early study.

FAPESP, the São Paulo Reserch Foundation joined the Giant Magellan Telescope as a founder member in December 2014 and subsequently Brazilian researchers and engineers became eager to contribute to the construction of the GMT first generation instruments. G-CLEF was at that time (and still is) the most advanced instrument (it has passed construction design phase review recently) and the principal investigator of this instrument, Dr. A. Szentgyorgyi suggested the study presented in this thesis, i.e. that we do lab experiments to test how adaptive optics may affect the efficiency of the instrument. The main goal of this project is then to describe an experiment in the lab that will test fibers, in terms of their throughput and stability, when fed by light in two different scenarios, with and without $\mathrm{AO}$ correction.

In paralell, Dr. Alexandre Mello, from the Federal University of Technology - Paraná, 
Brazil (UTFPR), was also studying a similar problem, but with simulations. We, then, started a collaboration with that group, on confirming the results of their simulations on optical bench experiments, and part of this thesis work went into a paper lead by Dr. Mello, published in Mello et al. (2018) and described in the following chapter of this thesis (chapter 3). 
Chapter 3

\section{Application of Adaptive Optics for Illumination Stability in Precision Radial Velocity Measurements in Astronomical Spectroscopy}

Monthly Notices of the Royal Astronomical Society 481, 3804-3809 (2018)

Accepted 2018 September 14. Received 2018 September 11; in original form 2018 May 15

Alexandre J.T.S. Mello, Antonin H. Bouchez, Andrew Szentgyorgyi, Marcos A. van Dam and Henrique Lupinari

This chapter is based on the results found by Mello et al. (2018), paper of which I am co-author. This paper was the result of a simulated-based study on how much the fiber illumination's stability responds to the insertion of an adaptive optics system into the observation. The instrument taken as model for the study was G-CLEF. The experimental counterpart for those simulations is our contribution for this work and was developed at IAG, in the context of this thesis.

\subsection{Illumination Stability Effects on PRV Measurements}

Usually AO is used to sharpen the imagery or reduce the required slit size of a spectrograph, but in the context of this work we are interested in the stability of the PSF, not necessarily in its sharpness.

Optical fibers are extremely persistent on the memory of the angle of incidence of the beam. So, ideally we would have the intensity profile well-centered within the fiber, as shown in the top panel of figure 3.1, which means that there is no preferential direction regarding the input optical axis of the fiber and therefore the average angle of incidence is 
zero. Nevertheless, when atmospheric turbulence is present, the PSF is not stable, causing it to move around the fiber. When it happens, as seen in the bottom panel of figure 3.1, the intensity ends up being decentered causing it to have a preferential direction and therefore the angle of incidence becomes different than zero, causing the fiber to produce an annulus pattern on the pupil, as imaged in figure 3.2.

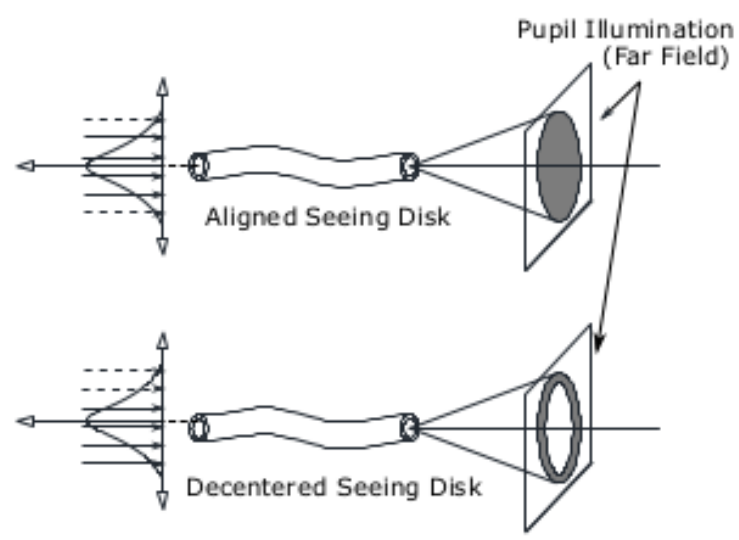

Figure 3.1: Illustration of misalignment effects on fiber's far field illumination. Dashed lines represents the light rays that does not enter the optical fiber. Top: Seeing disk aligned with fiber's optical axis, yielding a homogeneous far field illumination. Bottom: Seeing disk misaligned with fiber's optical axis, yielding a annulus pattern in the far field illumination. Figure 1 from Mello et al. (2018)

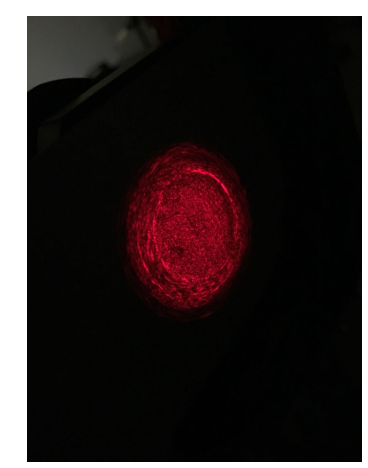

Figure 3.2: Annulus pattern in far field illumination yielded by a misaligned fiber in the lab. Experiment made in IAG lab.

As the input intensity profile moves, due to turbulence and consequently its centroid changes with respect to the fiber, it will cause the pupil to be illuminated in different shaped annuli, and uncalibrated and unpredictable aberrations will appear on the spectrograph detector.

Therefore, stabilizing the seeing disk on the optical fiber input would contribute to also stabilizing the output beam which would improve radial velocity precision. In addition, 
more light will enter the fiber, increasing throughput. AO systems, nowadays, are usually used to correct infrared observations and the ones on the optical passband do not reach the same image quality as the ones in IR, however they do concentrate visible light considerably to wavelength as low as $4000 \AA$.

\subsection{Adaptive Optics Stability Simulations}

YAO software package (Rigaut and van Dam (2013)) was used for this work. It is an open source AO Monte Carlo simulation tool. The atmospheric profile used in the simulations was based on a turbulence profiling of Cerro Las Campanas made by Goodwin et al. (2016). The objects on the simulation were stars of 6 and $12 \mathrm{mag}$, a typical bright and typical faint standard targets for G-CLEF also serving as guidestar, being observed through different atmospheric turbulence strengths. Results for $B$ band $(4400 \AA)$ and $I$ band $(7900 \AA)$ are shown in figure 3.3 .
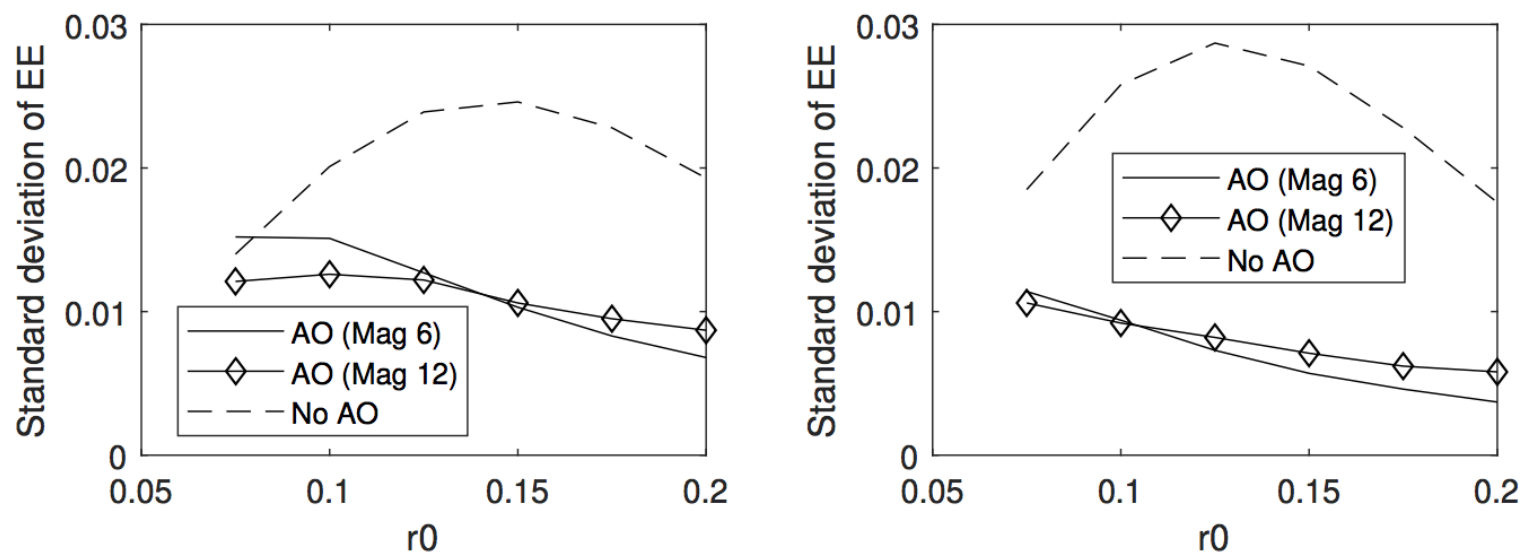

Figure 3.3: Standard deviation of encircled energy varying with turbulence strength. Dashed line represents the simulation without adaptive optics while continuum line the ones with AO correction with 6

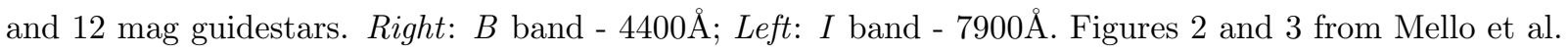
(2018).

Mello et al. (2018) found, as we can see in the figures, that only for very strong turbulence and short wavelength, AO does not reduce instability, while for all other cases it did show improvements on flux stability.

Simulations can be very precise and complex; however, they are not as reliable as experimental results. On the other hand, although the experiment is not as complete as the simulation, it is useful to improve confidence in the simulated results. Thus, these two 
complement each other and they are both crucial.

\subsection{Optical Bench Experiment}

In order to have compatible measurements in the optical bench, we designed a setup that mimics a small scale telescope; similar to the one used for other experiments of this thesis, described in detail in section 4.1.

The setup consists of a collimated $6350 \AA$ laser beam passing through a phase screen (described in section 1.3.1), which inserts the information of atmospheric turbulence correction, or the lack of it, into the system. This beam is focused into an optical fiber and then the fiber illuminates a detector, where the power emerging from the fiber is measured. In this case, differently from the other experiments done for this thesis, it was used a $300 \mu m$-diameter fiber and the low-order correction AO (GLAO). This difference in setup yielded, as expected, differences in the results measured.

The setup was envisioned to simulate an $8 m$ telescope observing through Las Campanas Observatory median seeing; which is not the whole GMT aperture but one of the primary segments. This still makes the analogy and study valid for reasons that will be explained later (section 4.1).

The data collected is simply the power detected by the powermeter over time, which translates into throughput. Flux stability is analyzed as the standard deviation of the measurements.

My measurements resulted in table 3.1, where throughput is calculated as the average power over time of each dataset and the beam stability is taken as the standard deviation of the measurements. Also pictured in figure 3.4.

Table 3.1 - Average fiber throughput depending on whether the observation is AO corrected or not.

\begin{tabular}{c||c} 
& Throughput $(\mu \mathrm{W})$ \\
\hline No correction & $309 \pm 13.6$ \\
AO corrected & $329 \pm 5.7$
\end{tabular}

In conclusion: GLAO correction yielded a 6\% (329/309) gain in throughput and increased flux stability by a factor of $2.4(13.6 / 5.7)$. 


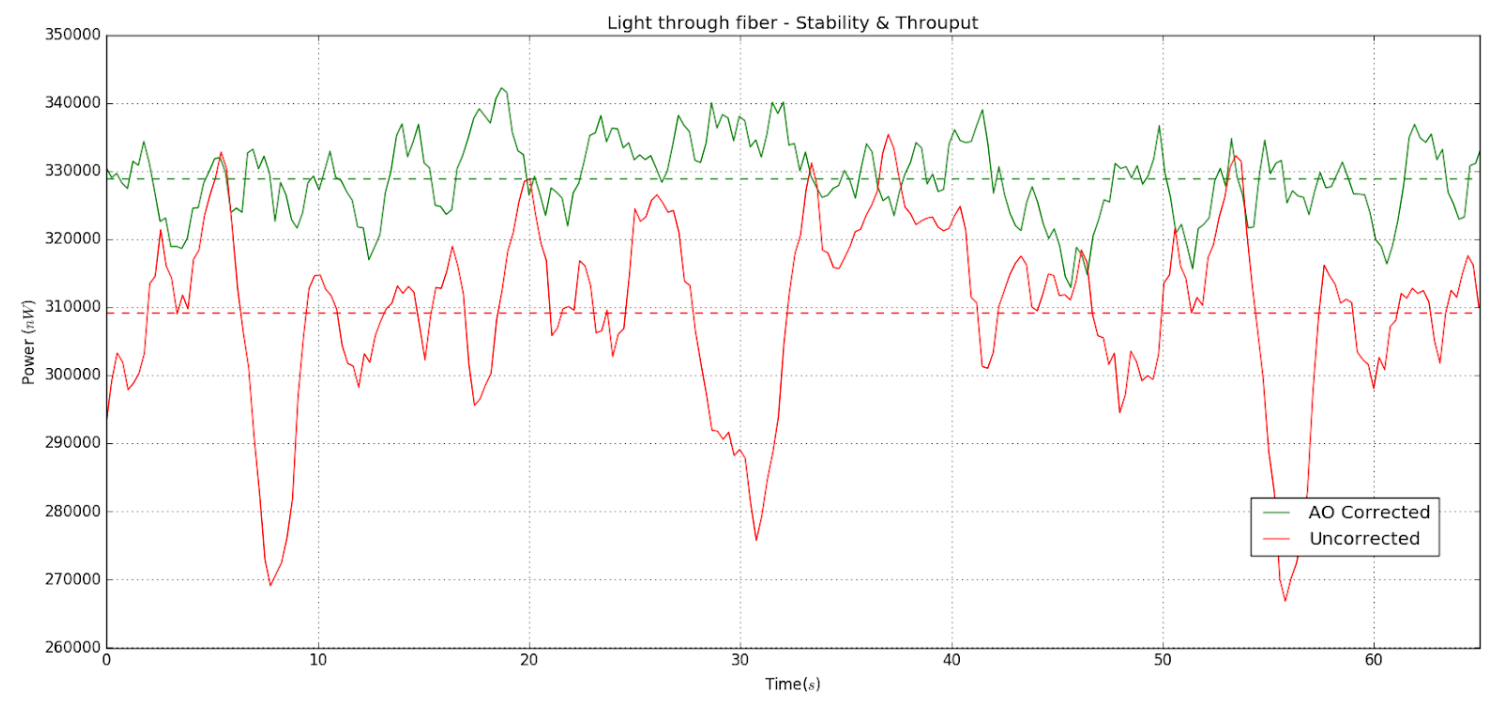

Figure 3.4: Fiber throughput power varying with time when subjected to AO correction (green line) and no correction (red line).

\subsection{Conclusion}

This chapter presented the idea of using adaptive optics to increase stability instead of just aiming for light concentration; and it did show improvements on the measurements. Simulations show no improvement only in extreme cases, of strong turbulence (seeing of $\sim 1.4$ ") and short wavelength ( $B$ band - $4400 \AA$ ). In particular, we have confirmed the increase of flux stability by a factor of 2.4 in the lab for a wavelength of $6350 \AA$. 
Chapter 4

\section{Optical Bench Experiments}

This chapter is dedicated to describing the setup construction for the main experiments described in this thesis, as well as the methods of measurement for each set of data.

The design and assembly of this setup started in my undergraduate research project in 2016, also advised by Professor Mendes de Oliveira and co-advised by Professor Steve Eikenberry. The goal, back then, was to create an optical bench setup capable of measuring optical fiber's focal ratio degradation, with the future intention of using it to test G-CLEF's real fiber under atmospheric turbulence and $\mathrm{AO}$ environments.

\subsection{Optical Bench Setup}

The idea is to simulate, on an optical bench, a point source being observed by one of the seven mirror segments of GMT feeding G-CLEF through a single mode, $100 \mu$ m-diameter, round fiber in two scenarios: one after being disrupted by atmosphere turbulence and the other after, in addition, being corrected by adaptive optics.

The choice for a $100 \mu \mathrm{m}$ round fiber is motivated by the fact that in precision radial velocity mode, each GMT mirror segment feeds a $100 \mu \mathrm{m}$ round G-CLEF optical fiber by pupil-slicing the beam, as explained in section 1.2.5. This is also the reason why only one of the segments is simulated on the bench. It is acceptable to carry out the study as such because each set of mirror segment and fiber is independent from the others and, therefore, by studying one of the sets, the results can be generalized to the whole aperture. In any case, it would not have been feasible to simulate the whole GMT aperture on the bench, mainly because the phase screen has a fixed value for the Fried parameter $\left(r_{o}=0.039 \mathrm{~mm}\right.$ @6300A), which sets a maximum size for the beam of $20 \mathrm{~mm}$ when a $60 \mathrm{~mm}$ beam size 
would have been needed.

The environment built for the experiment is described in the schematics of figure 4.1 below:
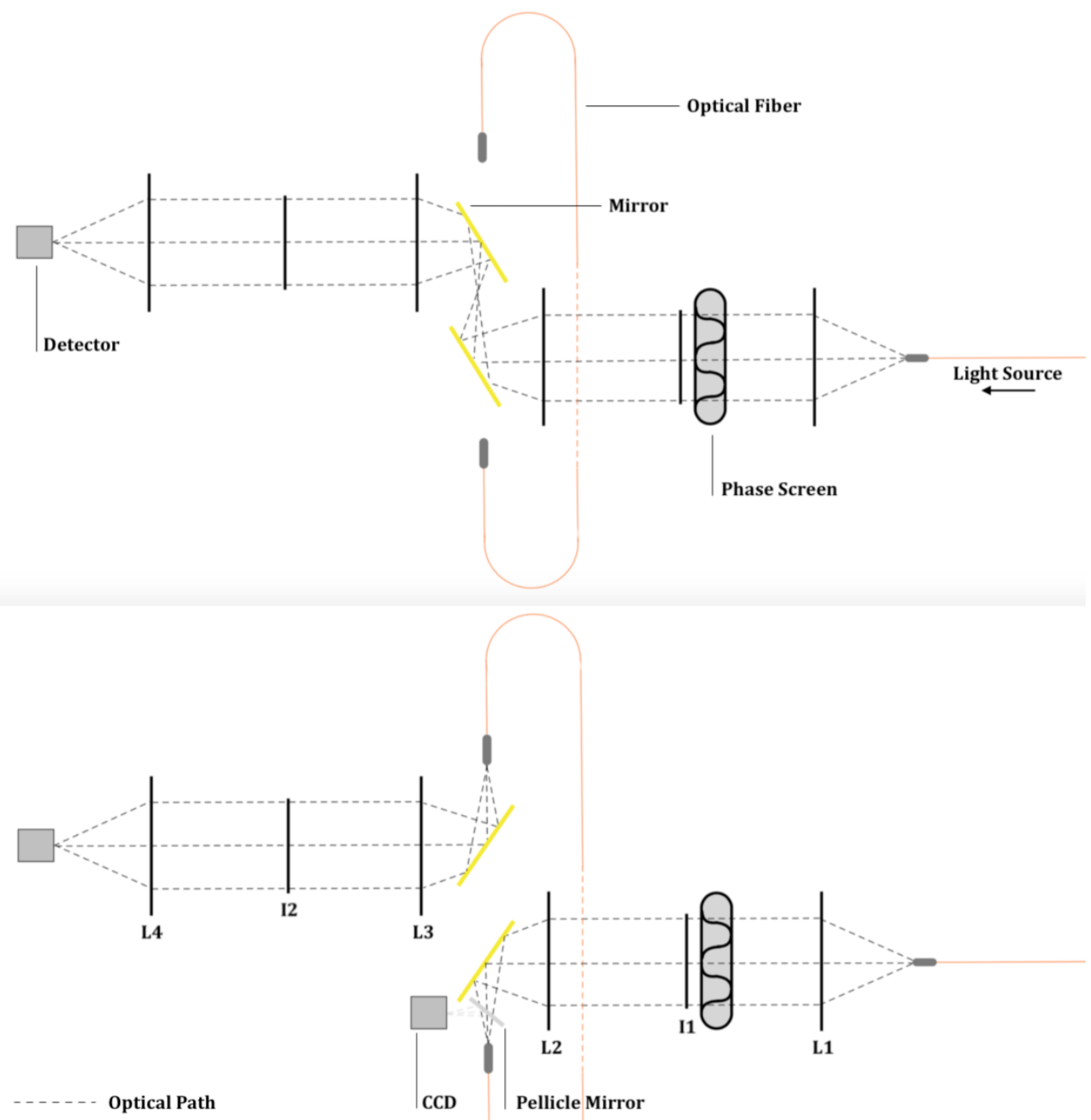

Figure 4.1: Schematics of optical bench setup; Top: Mirrors facing each other, allowing beam to transfer directly from first to second relay; Bottom: Mirrors facing the fiber ends, forcing the beam to go through the fiber in between relays.

A light source is placed on the right end of the bench; it is a halogen white source with a $633 \mathrm{~nm}$ filter. A band filter had to be used because the parameters on the phase screen are wavelength dependent, and we note that Lexitek's spreadsheet presents the parameters for that specific wavelength. 
The beam coming from the source gets collimated by the first lens (L1 in figure 4.1), therefore between the first and second lens a collimated space is formed. The phase screen, containing all the information regarding atmospheric turbulence and adaptive optics correction, is placed across this collimated space. The phase screen in this position is an analog to the atmosphere within the collimated space between the object being observed and the telescope collecting the light. Also in this space an iris is introduced (I1 in figure 4.1), to enable us to choose the desired beam diameter. This iris is set to an aperture of $20 \mathrm{~mm}$, in order to have the ratio between it and the turbulence cells on the phase screen be roughly the same as the one between the aperture of the GMT mirror and the turbulence cells of the atmosphere. Phase screens manufacturing details are given in section 1.3.1.

The collimated beam hits the second lens (L2 in figure 4.1), which represents one mirror segment of GMT, that focuses light. After focusing the light there are two possible paths: feeding the fiber or letting the beam go directly to the second lens relay. In between the two lens relays, there are two fold mirrors that are easily steerable by $90^{\circ}$ and the choice is made by turning the fold mirrors to face the fiber ends or to face each other. The position of the mirrors are secured by magnets, guaranteeing a precise positioning after steering them.

If the mirrors are facing each other, the light will come to a focus in between them; the beam will then spread again and will feed the second fiber relay (top panel in figure 4.1). The second relay represents G-CLEF. It is composed by a collimating lens, an iris, a focusing lens and a powermeter, in this order (L3, I2, L4 and detector in figure 4.1, respectively). The iris is set on this collimated space so that we are able to select the beam aperture that will reach the powermeter.

When the mirrors are facing the fiber ends, the first mirror will direct the focus to the fiber tip and the fiber will shed light onto the second lens relay. In this configuration, a pellicle mirror can be placed in front of the entrance fiber end, so that we can image the fiber tip with a CCD camera, in order to check alignment. Fiber ends stand on V-grooves placed over X-Y-Z-Tip-Tilt micrometer stages for a precise positioning and alignment.

All was mounted in a cage-like system, with off the shelf optical elements; the majority of them from ThorLabs. A picture of it is displayed in figure 4.2.

The alignment is done first by using the CCD camera that faces the input end on the fiber to roughly center the spot into the fiber tip. After that, with the light beam going 
Table 4.1 - Lenses used in the setup.

\begin{tabular}{l||c|c|c} 
Optical Element & $\phi(\mathrm{mm})$ & $f(\mathrm{~mm})$ & In figure 4.1 \\
\hline Collimating Lens & 50.8 & 100 & L1 \\
Focusing Lens & 25.4 & 60 & L2 \\
Collimating Lens & 50.8 & 100 & L3 \\
Focusing Lens & 50.8 & 100 & L4
\end{tabular}

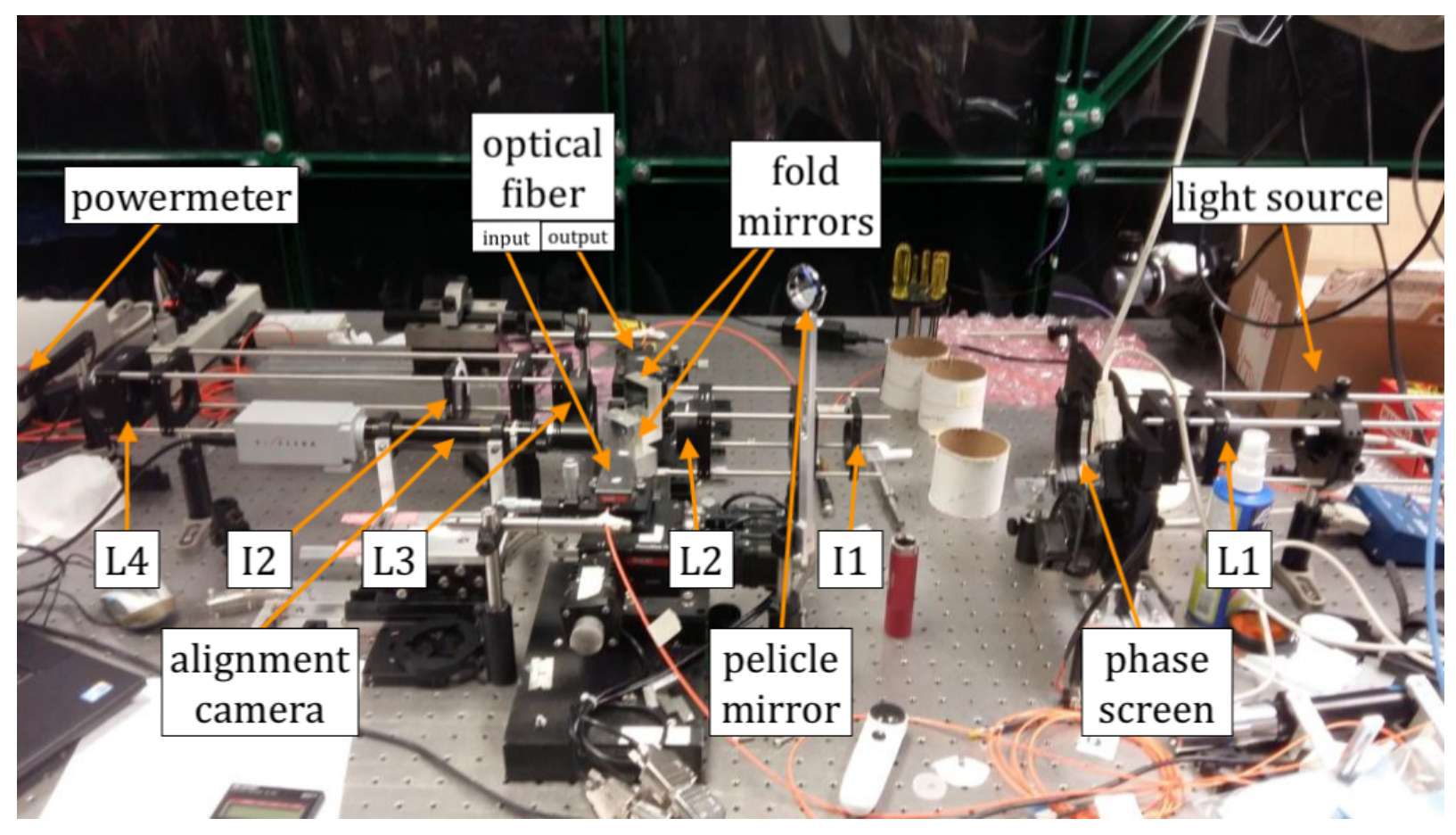

Figure 4.2: Picture of the setup used in the experiment.

through the fiber into the second relay and hitting the powermeter at the end, we optimize with the nobs in the fiber positioning stages in order to find the maximum power on the powermeter.

\subsubsection{Fiber Cleaving and Characterization Techniques}

One important step to assure that the experiment will be meaningful is to do fiber cleaving, ahead of its use in the experiment described below. This section then describes the treatment done to the fiber prior to experimental use.

First the fiber is cut, from a spool, on the desired length with a diamond tip razor (ThorLabs S90R). With this cut, the ends of the fiber will be shattered. In order to make the ends more flat, we cleave them a second time, but now with more precise tools, described in the following. 
In order to cleave the ends in a way that the glass does not shatters we need to remove the polymer (polyamide) envelope from it. We do so by using a specific machine (PWS; 3sae Technologies, Inc.) that removes this envelope, by heating it up with plasma, evenly from all sides. It also can be done with a lighter, but in this case the fiber will not be heated evenly, and it may cause it to deform.

After having the envelope removed from the core, it is ready to be cleaved. There are two ways to do so. The first one, used for fibers with core diameter smaller than $150 \mu m$, with a commercial 'common' cleaver (Fitel S326) and the second one with a more sophisticated cleaver (LCC II; 3sae Tchnologies, Inc.), used for fibers with core diameter larger than $150 \mu m$, the latter applies a certain tension on the fiber before cleaving it, in order to have a better final surface. We used the first process, which resulted in a better fiber surface outcome since we are using $100 \mu \mathrm{m}$ fibers.

After having cleaved the fiber we have to verify if the process was successful, and we do so by imaging the fiber ends with an interferometer (Fibo 300 Interferometer), shown in appendix A (two fibers are shown in the appendix, 'fiber 2' was the one used on the experiments).

\subsection{Measurements}

In this section we describe the two main measurements that will be done in the context of this work, focal ratio degradation described in subsection 4.2.1 and throughput and stability described in subsection 4.2.2

There are six possible configurations for measurements. Three of the options are: (1) not using any phase screen, (2) using phase screen without AO correction and (3) using phase screen with AO correction. For each of the above options, we then put the fiber in the optical path or not. Measurements without the use of a fiber are used for calibration. It is taken as the base for normalizing the other measurements.

In all measurements that contain any phase screen in the optical path, we make sure that the phase screen moves during the measurements, in order to better imitate atmospheric turbulence. A circular motor holds it and spins it with the desired frequency.

A quick experiment was done to define how fast and how many spins the phase screen should complete for each measurement. This experiment consisted on imaging the beam 
with the CCD that is meant for allignment of the fiber input, with different spin speeds and total number of spins. We concluded that it actually does not matter, as long as at least one full spin is done during the measurement.

\subsubsection{Focal Ratio Degradation}

For the focal ratio degradation experiment the idea is to trace the beam profile coming out of the fiber. It is done by measuring how much energy is contained on each radius of the beam. As explained in section 1.4.1, FRD is an effect that the beam suffers after going through an optical fiber where the output beam has a greater spread angle than the input beam.

The iris placed across the second collimated space (I2) is the one responsible for selecting the radius of the beam we intend to measure. The measurement starts with the iris fully open $(\mathrm{R} \rightarrow \infty)$, letting all the light reach the powermeter. The phase screen completes a full spin and as soon as it stops, the powermeter is shined by a regular flashlight. In the cases that there is no phase screen, the time collecting data is still the time it would take for the phase screen to complete a full spin, for fairness.

While the flashlight is on and aiming at the powermeter, the next iris radius is adjusted, smaller every time. When the iris is set, the flashlight is turned off and the phase screen motor is triggered to start spinning again. When it ends, the flashlight is turned on again, iris ajusted and so on and so forth, until we get to an iris size $R=0$, in which we measure the background, since, in this case, no light from the system is reaching the powermeter. After the iris is fully closed, there is an additional step with the iris fully open (once again), to check how stable the source is, along the time of the data taking.

The whole process described above happens with the powermeter collecting data, nonstop, including the times when the flashlight is on. The flashlight actually serves to flag where exactly the data we are interested in start and where they end.

An example of how the raw data are arranged is displayed in figure 4.3. Each step in figure 4.3 bottom panel represents the power collected for a specific iris aperture.

This process is repeated for three of the six configurations, the three configurations that make use of the fiber in the optical path. 

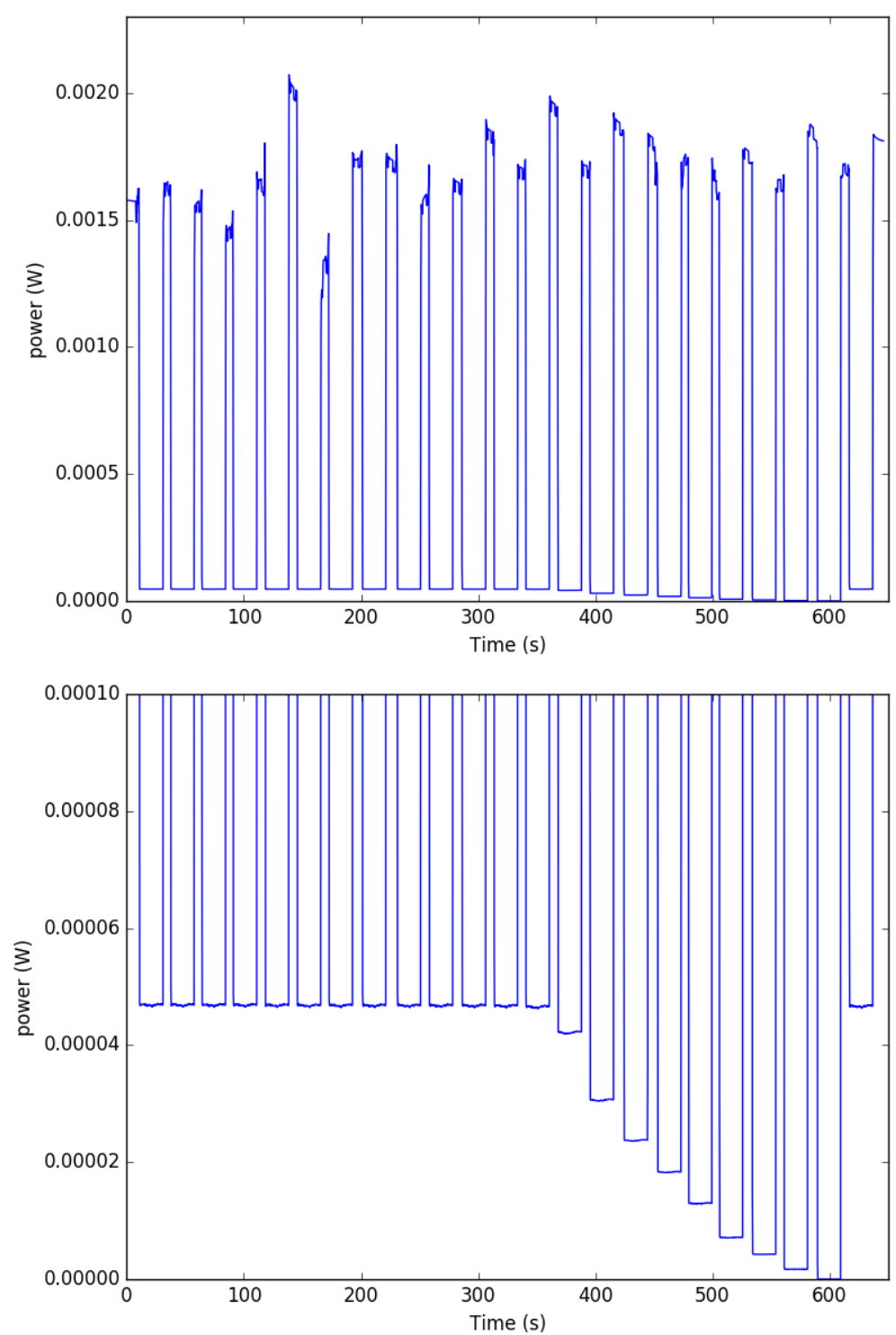

Figure 4.3: Top: Raw data of one set of measurement for FRD experiment. Bottom: Zoom in to show data of interest.

\subsubsection{Throughput and Stability}

The measurement for throughput and flux stability is very similar to the FRD measurement, but simpler. Instead of getting values for power for every iris aperture to trace a profile, in this case, our interest is to study only one aperture. The aperture chosen was $F / 3$. This choice of beam speed is made to take into account some light loss for which FRD is usually responsible, in fiber-fed instruments. The choice will be clear in chapter 5, when we explain the analysis and results of the FRD experiment. 


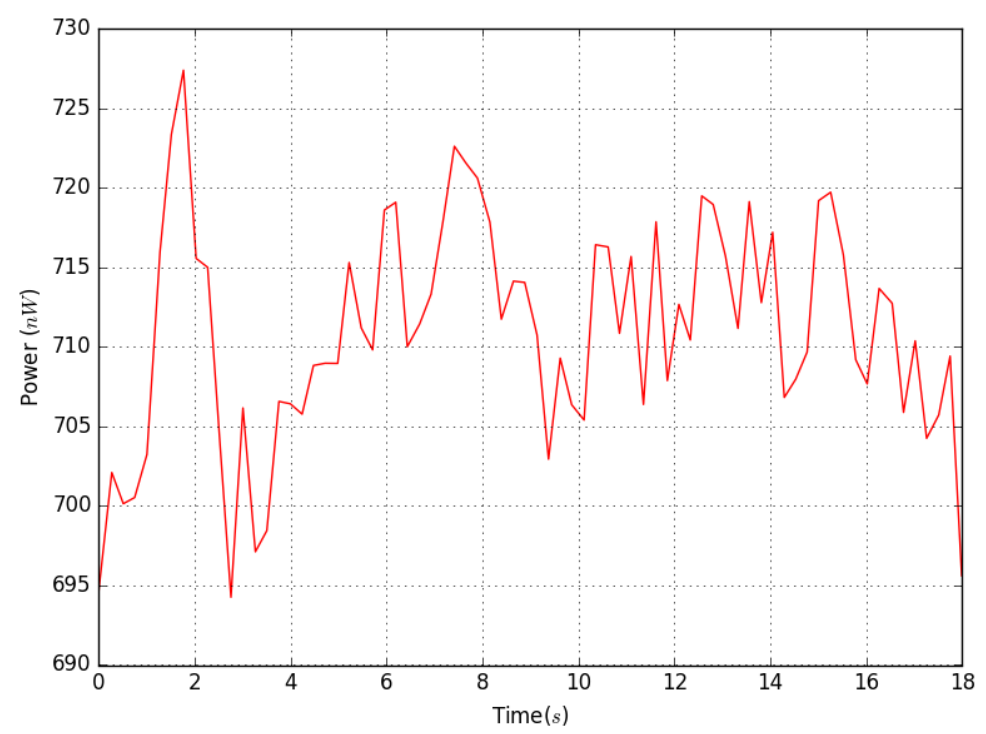

Figure 4.4: Example of raw data of power vs time for throughput and stability measurements.

The experiment for measuring throughput and flux stability uses 10 full spins of the phase screen when doing measurements, and they are repeated for each of the six configurations of the setup. The powermeter was collecting data every $0.03 \mathrm{~s}$. The 10 spin cycle was chosen arbitrarily.

An example of how the raw data are arranged is displayed in figure 4.4, and the analysis of all the collected data is in chapter 5 . 
Chapter 5

\section{Analysis and Results}

In this chapter, we present the results of the analysis of the data collected for the experiments described in chapter 4, using python routines developed during the course of this work.

\subsection{Focal Ratio Degradation}

Our goal with this experiment, described in section 4.2.1, was to determine whether or not the use of adaptive optics would help decrease light loss caused by focal ratio degradation.

The first step in the analysis is to identify in the raw data, pictured in figure 4.3, where the meaningful datapoints are located. The use of a flashlight, described in section 4.2.1, was for this purpose. Its strong signal differs drastically from the level of the actual signal we are measuring, enabling us to flag where the steps containing interesting data start and where they end.

We created a python routine that detects very sudden changes in power over time. In order for it to work, it is necessary to define a value for power $_{o}$, a constant, defined arbitrarily as half the height of the lowest peak. With this simple step, we guarantee that the queries made with equation 5.1 will not return false positives:

$$
\mid \Delta \text { power } \mid>\text { power }_{o}
$$

Where:

$$
\Delta \text { power }=\text { power }_{t 1}-\text { power }_{t 2}
$$


And power $_{t}$ is the power measured in time $t$ and $t 2>t 1$. The program analyses the set of data over time, and when it detects a negative variation in power (i.e. power $_{t 1}>$ power $_{t 2}$ ) higher in absolute value than power $_{o}$ (Eq. 5.1), it interprets it as the start of a step of useful data. Similarly, when it detects a positive variation in power (i.e. power $_{t 1}<$ power $_{t 2}$ ) higher in absolute value than power $_{o}$ (Eq. 5.1), it interprets as the end of it. Start and end are shown in figure 5.1 as green dots and red squares respectively. They yield the time coordinates where the data can be found.

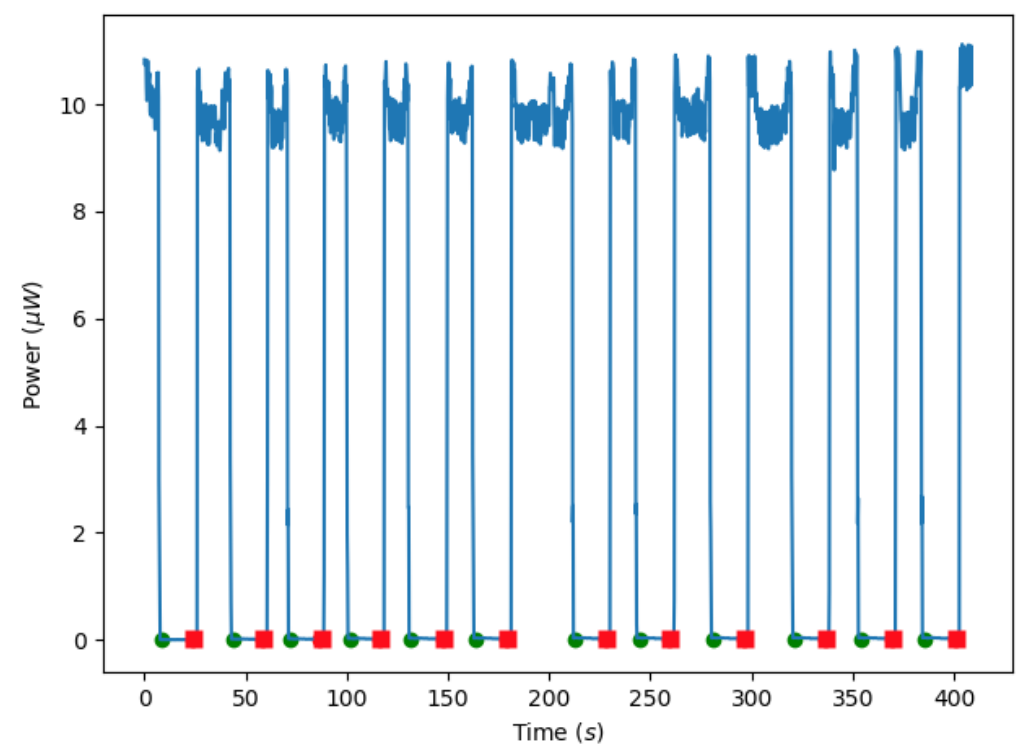

Figure 5.1: Dataset for our FRD experiment: shown in green dots are the time coordinates of the starting points of useful data and in red squares the coordinates for the ends.

We, then, have time coordinates that flag where the data for each iris aperture is contained. Now we can calculate the average power in each step and assign it to the respective iris aperture. For each aperture we assign also an f-number, which is simply:

$$
F / \#=f / D
$$

Where $f$ is the focal length of the collimating lens (L3 in the scheme of figure 4.1) and $D$ is the iris aperture. The beam profile can then be traced in three different setup configurations: a diffraction limited system (no phase screen), a system disrupted by atmospheric turbulence and a system corrected by AO, each of them feeding a $100 \mu m$ fiber at $F / 3$. The beam profile coming from the fiber in each of the cases is shown in the plot pictured in figure 5.2 . 


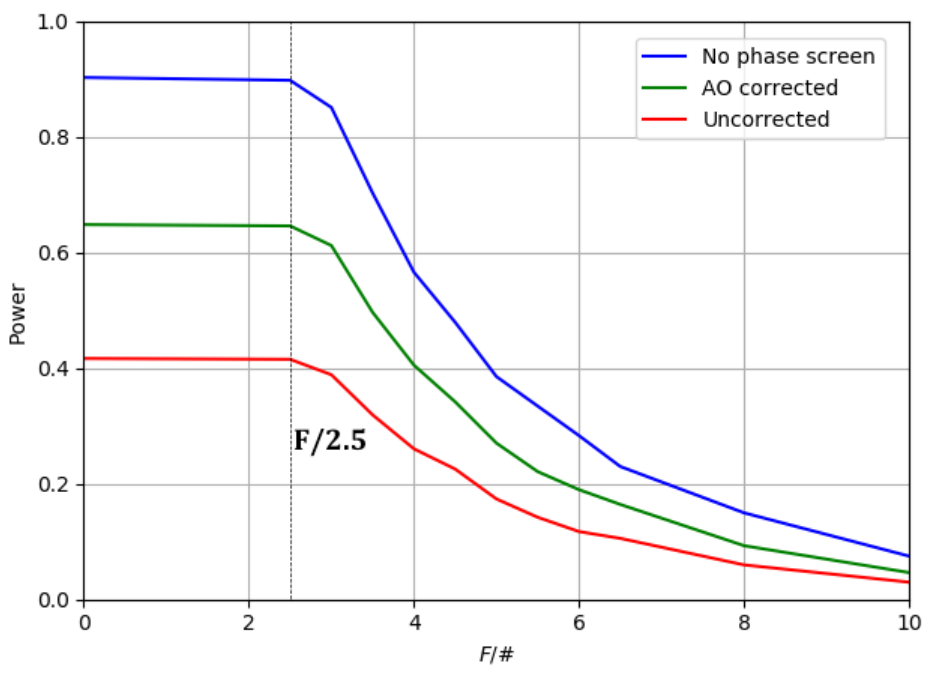

Figure 5.2: Focal ratio profile of the beam in three different environments with fiber.

The gain in overall throughput shall not be taken into account in this experiment, given that no normalization is done and this is not the goal of this experiment. This will be addressed in the next experiment, described in section 5.2 .

We measured in all three different configurations an $F / 2.5$ beam coming from the fiber, which were fed by an $F / 3$ beam, regardless of the use of adaptive optics or not. In fact even with no phase screen on the optical path the fiber still presents a similar profile. It was because of the $F / 2.5$ beam profile coming from the fiber that we decided to have the beam speed of the next experiment as $F / 3$, to account for a portion of light loss that comes from the FRD problem.

\subsection{Throughput and Stability}

The goal of the last experiment was to study the beam profile coming out of the fiber and analyze if AO was able to decrease FRD losses. As for this second experiment, the goal is to study how much the efficiency of G-CLEF, in terms of total throughput and flux stability, would improve with the use of adaptive optics, when compared with the seeing limited case. This is the same kind of measurement as the one done for Mello et al. (2018), described in chapter 3. The main difference between them was the diameter of the fiber tested and the type of $\mathrm{AO}$ correction. The measurements done for the paper were done using a $300 \mu m$-diameter fiber and GLAO correction, whereas for the following experiment 
it was used a $100 \mu m$-diameter fiber and NGSAO correction (higher correction order when compared with GLAO).

In contrast to the FRD measurements, this experiment does not need a code to identify pieces of data in which the important information is contained. For each of the configurations there is a separate set of data in which there is only continuous and useful information, as imaged in figure 4.4. All the raw data of this experiment are shown in table 5.1 and plotted in figure 5.3 together with the background noise. The data collected are power over time and the results displayed in the tables are the mean power and the standard deviation of that measurement for each case. The background noise was assigned as the mean and standard deviation of the measurement done with the system working with the iris (I2 in figure 4.1) fully shut, in order to detect only signal coming from outside of the experiment.

Table 5.1 - Information of throughput and stability for each of the six configurations; ploted in figure 5.3

\begin{tabular}{c||c|c|c} 
& No PS & AO Corr. & Uncorr. \\
\hline \hline No Fiber $(n W)$ & $142.18 \pm 1.23$ & $140.73 \pm 1.24$ & $138.47 \pm 1.66$ \\
\hline With Fiber $(n W)$ & $109.77 \pm 1.26$ & $76.51 \pm 1.47$ & $47.24 \pm 3.63$
\end{tabular}

In this experiment it is important also to take measurements with the three setups that do not make use of the optical fiber because they are used to make the normalization of throughput. Since our analysis is focused on comparing how AO correction affects the overall system efficiency, it is crucial to be able to differentiate other sources of error.

It was acknowledged that the two phase screens have slightly different opacities in the wavelength used (as can be seen from the three top lines of figure 5.3 and from the 'no fiber' line in table 5.1 ), by about $1.0 \%$ and $2.6 \%$ for $\mathrm{AO}$ and no $\mathrm{AO}$ phase screens, respectively. These differences were compensated by normalizing the 'with fiber' power measurements with the respective 'without fiber' mean power. The resulting measurement is then the amount of light that was actually reaching the fiber.

After normalizing the measurements we got the results in table 5.2 and the comparative plot shown in figure 5.4. The throughput is calculated as the average power over time of each dataset. The beam stability is taken as the standard deviation of the measurements.

It is very clear, by looking at figure 5.4, that the line that represents the measurements without any phase screens and the noise shows a beating, very well defined in frequency, 


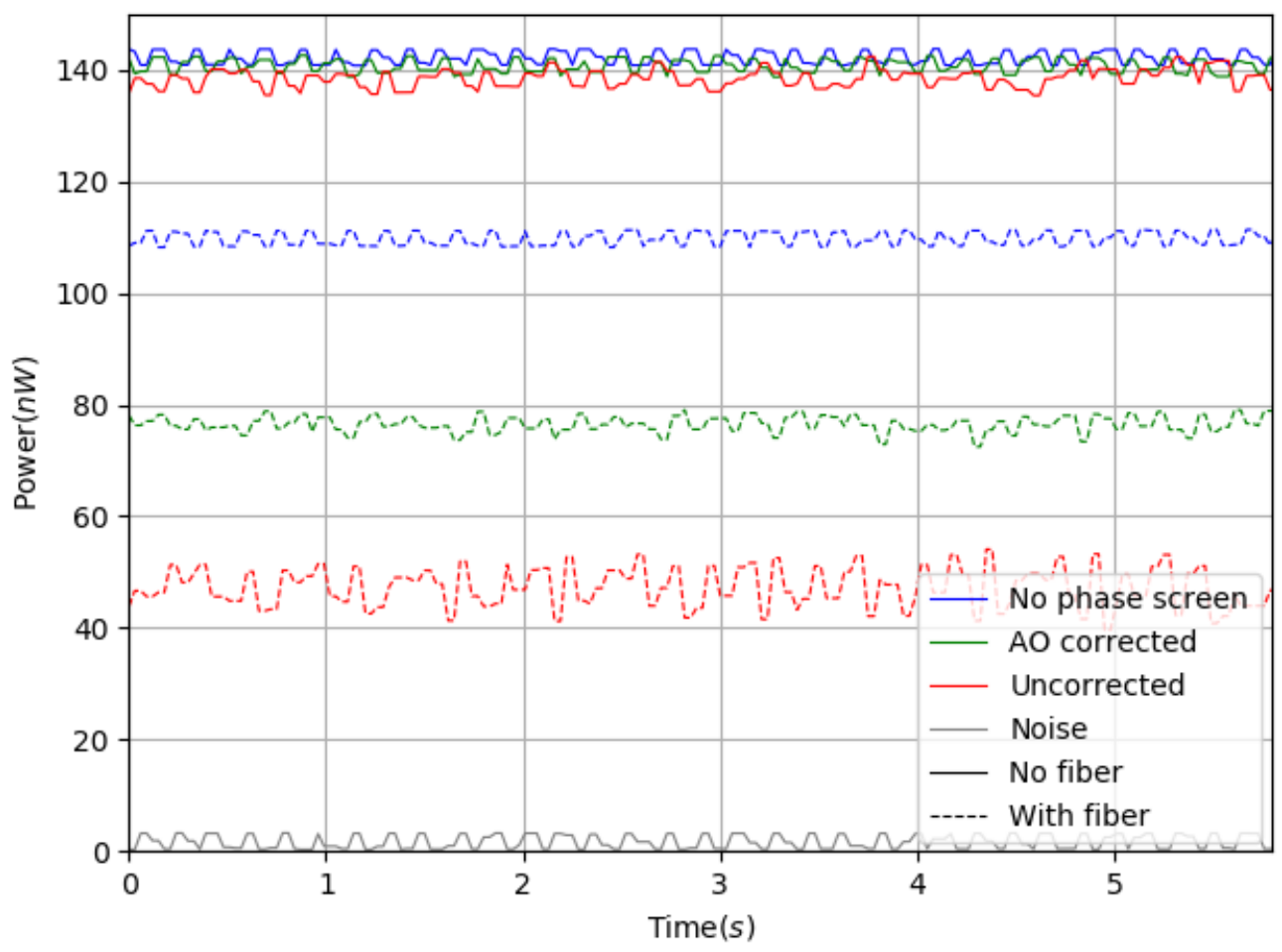

Figure 5.3: Power over time of each of the six configurations and noise curve. Raw data.

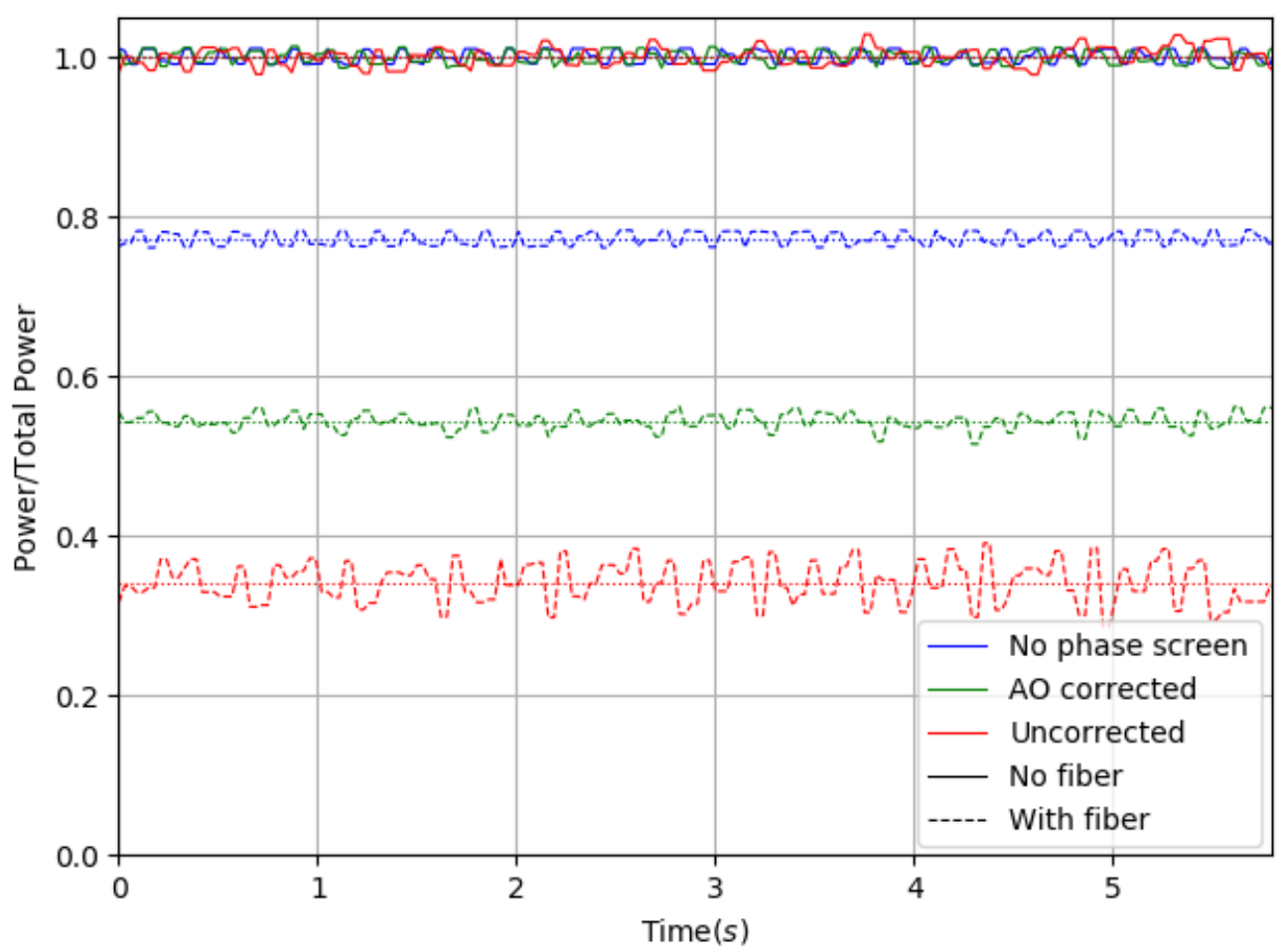

Figure 5.4: Normalized throughput over time for each of the six configurations. 
Table 5.2 - Normalized stability and throughput for each of the six configurations.

\begin{tabular}{c||c|c|c} 
& No PS & AO Corr. & Uncorr. \\
\hline \hline No Fiber & $100.00 \pm 0.87$ & $100.00 \pm 0.88$ & $100.00 \pm 1.20$ \\
\hline With Fiber & $77.21 \pm 0.88$ & $54.37 \pm 1.05$ & $34.11 \pm 2.61$
\end{tabular}

about $6 \mathrm{~Hz}$. This was not expected. This configurations should yield almost "perfect" stability since there is nothing disruptive (phase screens or other obvious sources of error) in the optical path. We then concluded that this intrinsic instability could also be affecting the measurements with phase screens. This is less than ideal, given that we want to compute the effects caused solely from the insertion of $\mathrm{AO}$ into the system. To be sure that this beating is also affecting the other measurements, we did a Fourier transform of the data from the time domain to frequency domain. It resulted in the plot shown in figure 5.5 , confirming our suspicion.

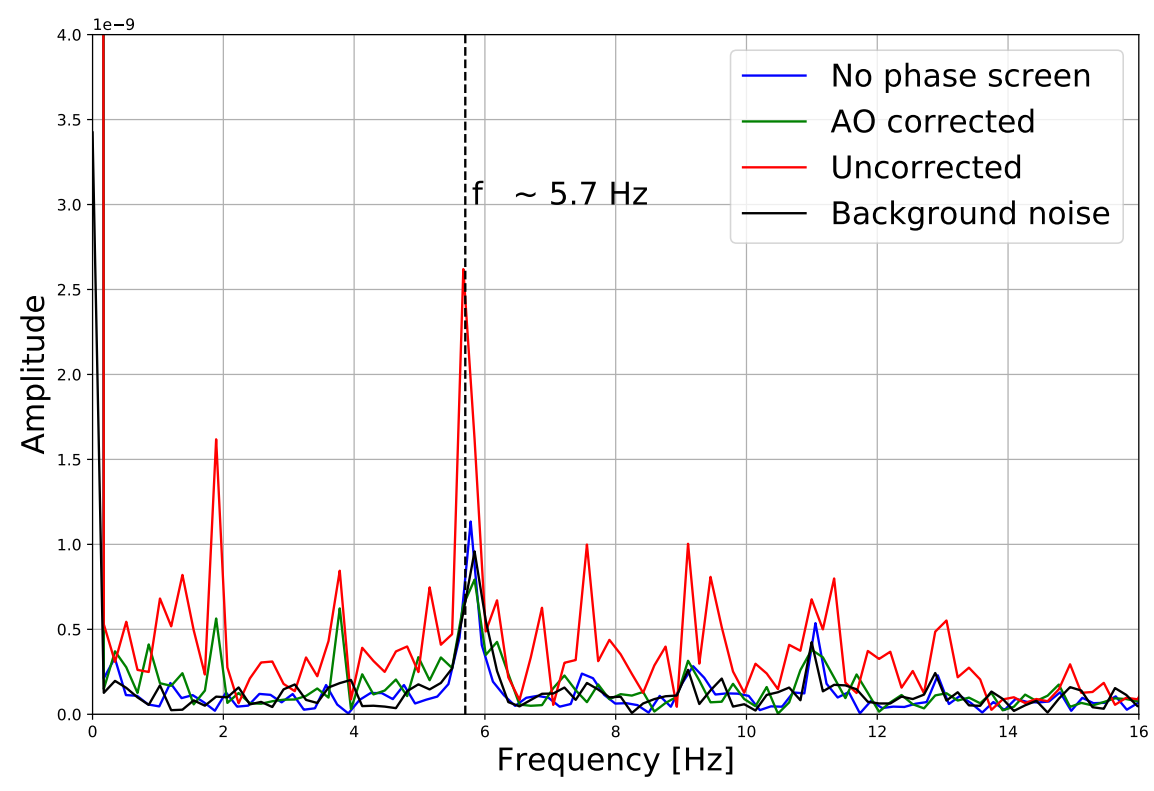

Figure 5.5: Fourier transform of the data taken using the three configurations that use the optical fiber.

The very prominent peak in $5.7 \mathrm{~Hz}$ is the identified beating. We can see on the "no phase screen" line (blue line in figure 5.5) that this peak is the highest and one of the few for that configuration. Also we can see that it is the most prominent in all configurations, which means that the beating is indeed intrinsic to the setup and affects equally all the measurements. We can also see a second peak around $11 \mathrm{~Hz}$, which is most probably a harmonic of the $5.7 \mathrm{~Hz}$ peak. 
It is not very clear to us where this beating is coming from. It could be a flicker on the light source, an external source of error or due to mechanical vibrations coming from the phase screen holder motor. However, this is not likely to influence our results given that it is present in all three configurations.

We can then conclude that the error measured, shown in table 5.2 , is also due to a setup deviation and not a part of the experiment itself. The total standard deviation is then shown in equation 5.4.

$$
\sigma_{P S m}^{2}=\sigma_{\text {Setup }}^{2}+\sigma_{P S r}^{2}
$$

Where $\sigma_{P S m}$ is the standard deviation measured in each of the configurations, $\sigma_{\text {Setup }}$ is the error related to the intrinsic beating and $\sigma_{P S r}$ is the real error coming from the phase screen. We find the results displayed in table 5.3.

Table 5.3 - Information on stability and throughput after taking into account the presence of an intrinsic beating in the setup.

\begin{tabular}{c||c|c} 
& AO Corr. & Uncorr. \\
\hline \hline With Fiber & $54.37 \pm 0.56$ & $34.11 \pm 2.46$
\end{tabular}

In order to be able to compare flux stabilities between the setups we can not use only the value of the standard deviation, but we rather use the signal-to-noise ratio of each configuration. Signal-to-noise $(\mathrm{S} / \mathrm{N})$ is here defined as the ratio of the mean throughput measured over its respective standard deviation. When using AO we got 97.09 of $\mathrm{S} / \mathrm{N}$ while without it we only get 13.87 , a factor of 7 difference in flux stability. As for throughput, the use of AO yielded an increase of $60 \%$ in overall power collected. This value was calculated from the ratio between the throughput in the AO setup and the one on the setup without AO (54.37/34.11; table 5.3). 
Chapter 6

\section{Discussion, Conclusion and Prospects}

We study what are the effects of having adaptive optics for the efficiency of G-CLEF, a fiber-fed, seeing limited echelle spectrograph to be installed on GMT at first light. Experiments were developed to tackle the subject in two different fronts: (1) how the AO system would decrease focal ratio degradation effect on the beam and (2) how AO would improve the instrument throughput and flux stability.

For the FRD experiment we concluded that the focal ratio of the beam that comes out of the fiber is not dependent on the use of adaptive optics. Even the diffraction limited case yields a beam profile similar to the one from a seeing limited scenario. Therefore, not even an AO system with strehl ratio of 1 would minimize FRD effects on the beam coming out of the fiber.

The second experiment, that addressed the throughput and stability measurements, was done in order to investigate the gain that adaptive optics would bring on those matters. We found an increase in flux stability of a factor of 7 and an increase in throughput of $60 \%$ due to the use of $\mathrm{AO}$.

A factor of seven increase in flux stability is not quite significant on the precision level. We were not able to deduce a direct relation between the beam stability measured and the precision that the instrument would be able to achieve. Something to be considered is that, after the beam goes through the double scrambler, it is desensitized by a factor of 3,500. Therefore, micro changes in temperature, drifting in the detector, scatterlight and micro vibrations from the movement of the telescope, for example, become more significant sources of error than the beam instability. However, we can still argue that, since AO is stabilizing the beam, it might still bring some, even if minimal, improvement to the precision. 
The results showed that the use of an AO system would bring an increase of $60 \%$ in throughput for G-CLEF, when working on precision radial velocity mode. This result is of the order of magnitude expected, as previously discussed in chapter 2. If AO would be used in the first phase of GMT operations, when only 4 of the 7 mirror segments are installed, it would collect almost as much light as if the telescope was working at full capability, with all the mirrors and no AO. At the time that this dissertation is being written (August 2019) the GMT project is not expected to deliver AO capabilities in its early phase (except for GLAO), as was mentioned in section 1.2. However, if the United States National Science Foundation (NSF) comes up with new funding for the project, in the next years, perhaps the adaptive optics facility will be delivered earlier than originally expected.

Considering the equation for comparison between magnitudes and fluxes:

$$
\Delta m a g=-2.5 \log _{10}\left(\frac{F_{s}}{F_{A O}}\right)
$$

Where $\triangle m a g$ is the range of magnitude that AO will enable G-CLEF to observe, since in the seeing limited case it collects a flux of $F_{s}$ and with $\mathrm{AO}$ it will collect $F_{A O}$, which is $1.6 F_{s}$. Therefore we can conclude that G-CLEF will be sensitive to objects $0.5 \mathrm{mag}$ fainter when using AO on the observations. The faintest magnitude G-CLEF is designed to observe, in precision radial velocity mode, is between 10 and $11 \mathrm{mag}$, depending on the signal-to-noise ratio required for the observation. Due to this increase in range, G-CLEF will have several new stars to target and look for signs of exoplanets.

Besides, for the objects that are already suitable targets for G-CLEF, the exposure time necessary to make an observations using AO would be $38 \%$ shorter when compared with the seeing limited case.

At last, it is also interesting to point out that, with $\mathrm{AO}$ enabling $60 \%$ more light into the system the peak throughput of G-CLEF, at $6550 \AA$, would be $9.44 \%$ (60\% increase in numbers from table 1.3) in PRV mode, almost reaching the throughput desired for the High Throughput observation mode, 11.46\% (table 1.3).

This work concluded that the use of adaptive optics together with G-CLEF can bring a significant boost in efficiency for the instrument. If $\mathrm{AO}$ would to be installed in the early phase of GMT, it would not only increase G-CLEF's efficiency, but also help GMT's team collecting phasing data, to better understand how the new telescope works. 


\subsection{Prospects}

This work can be further improved. We plan to make changes in the experimental setup and refine the method used for doing the measurements. In order to be able to measure spectrograph stability, instead of only flux stability, as was measured in this work, the detector has to be changed, from a powermeter to a CCD camera. In this new future experiment we will not study the changes in power of the beam, but rather compare the near-field and far-field stability of the fiber face and output beam respectively, with and without AO. The idea is to study how the centroid of the PSF of the beam coming out of the fiber behaves when the fiber is fed with an AO system compared with the case without AO. These measurements will give us the spectral stability enabled by AO. However, in order for the experiment to be complete and worth publishing (together with the work presented in this dissertation), we will have to do these measurements using G-CLEF double scrambler on the optical system, and that will not be available until 2020/2021 when the double scrambler will be prototyped. 


\section{Bibliography}

Anglada-Escudé G., Amado P. J., Barnes J., Berdiñas Z. M., Butler R. P., Coleman G. A. L., de La Cueva I., Dreizler S., Endl M., Giesers B., Jeffers S. V., Jenkins J. S., Jones H. R. A., Kiraga M., Kürster M., López-González M. J., Marvin C. J., Morales N., Morin J., A terrestrial planet candidate in a temperate orbit around Proxima Centauri, Nature, 2016, vol. 536, p. 437

Avila G., FRD and scrambling properties of recent non-circular fibres, Proc. SPIE, 2012, vol. 8446

Barden S. C., Review of fiber optic properties for astronomical spectroscopy, Proc. SPIE, 1995, vol. 2476

Barden S. C., Review of Fiber-Optic Properties for Astronomical Spectroscopy, ASP Conference Series, 1998, vol. 152, p. 14

Belland B., Gunn J., Reiley D., Cohen J., Kirby E., Cesar de Oliveira A., Souza de Oliveira L., Roberts M., Seiffert M., Focal Ratio Degradation for Fiber Positioner Operation in Astronomical Spectrographs, Journal of Astronomical Instrumentation, 2019, vol. 8, p. 1950007

Bely P. Y., Chapter 1: Astronomical Observations In The Design and Construction of Large Optical Telescopes, Astronomy and Astrophysics Library, Ed. Springer, 2003

Boss A. P., Butler R. P., Hubbard W. B., Ianna P. A., Kürster M., Lissauer J. J., Mayor M., Meech K. J., Mignard F., Penny A. J., Quirrenbach A., Tarter J. C., Vidal-Madjar A., Working Group on Extrasolar Planets, IAU Transactions, Reports on Astronomy 2002-2005, 2007, vol. 26A, p. 183 
Cassan A., Kubas D., Beaulieu J. P., Dominik M., Horne K., Greenhill J., Wambsganss J., Menzies J., Williams A., Jørgensen U. G., Udalski A., Bennett D. P., Albrow M. D., Batista V., Brillant S., Caldwell J. A. R., Cole A., Coutures C., Cook K. H., Dieters S. Dominis Prester D., One or more bound planets per Milky Way star from microlensing observations, Nature, 2012, vol. 481, p. 167

Fried D. L., Optical Resolution Through a Randomly Inhomogeneous Medium for Very Long and Very Short Exposures, Journal of the Optical Society of America, 1966 , vol. 56 , p. 1372

Gillon M., Triaud A. H. M. J., Demory B.-O., Jehin E., Agol E., Deck K. M., Lederer S. M., de Wit J., Burdanov A., Ingalls J. G., Bolmont E., Leconte J., Raymond S. N., Selsis F., Turbet M., Barkaoui K., Burgasser A., Burleigh M. R., Carey S. J., Chaushev A., Copperwheat C. M., Delrez L., Fernandes C. S., Seven temperate terrestrial planets around the nearby ultracool dwarf star TRAPPIST-1, Nature, 2017, vol. 542 , p. 456

Goodwin M., Jenkins C., Lambert A., Generalized Slope Detection and Ranging turbulence profiling at Las Campanas Observatory, Monthly Notices of the Royal Astronomical Society, 2016, vol. 461, p. 1701

Halverson S., Roy A., Mahadevan S., Ramsey L., Levi E., Schwab C., Hearty F., MacDonald N., An Efficient, Compact, and Versatile Fiber Double Scrambler for High Precision Radial Velocity Instruments, The Astrophysical Journal, 2015, vol. 806 , p. 61

Hubin N., Arsenault R., Conzelmann R., Delabre B., Le Louarn M., Stroebele S., Stuik R., Ground Layer Adaptive Optics, Comptes Rendus - Physique, 2005, vol. 6, p. 1099

Mayor M., Queloz D., A Jupiter-mass companion to a solar-type star, Nature, 1995, vol. 378 , p. 355

Mello A. J. T. S., Bouchez A. H., Szentgyorgyi A., van Dam M. A., Lupinari H., Application of adaptive optics for illumination stability in precision radial velocity 
measurements in astronomical spectroscopy, Monthly Notices of the Royal Astronomical Society, 2018, vol. 481, p. 3804

Parry I. A., The Astronomical Uses of Optical Fibers, ASP Conference Series, 1998, vol. 152 , p. 3

Podgorski W., Thoughput Error Budget Report; Doc.\#:SAO-INST-REF-00025, 2018

Rigaut F., van Dam M., Simulating Astronomical Adaptive Optics Systems Using Yao, Proceedings of the Third AO4ELT Conference, 2013

Rodler F., López-Morales M., Feasibility Studies for the Detection of $\mathrm{O}_{2}$ in an Earth-like Exoplanet, ApJ, 2014, vol. 781, p. 12

Szentgyorgyi A., Baldwin D., Barnes S., Bean J., Ben-Ami S., Brennan P., Budynkiewicz J., Catropa D., Chun M.-Y., Conroy C., Contos A., Crane J. D., Durusky D., Epps H., Evans I., Evans J., Fishman V., Frebel A., Gauron T., Guzman D., Hare T., Jang B.-H., Jang J.-G., Jordan A., The GMT-consortium large earth finder (G-CLEF): an optical echelle spectrograph for the Giant Magellan Telescope (GMT), Proc. SPIE, 2018, vol. 10702

Szentgyorgyi A., Baldwin D., Barnes S., Bean J., Ben-Ami S., Brennan P., Budynkiewicz J., Chun M.-Y., Conroy C., Crane J. D., Epps H., Evans I., Evans J., Foster J., Frebel A., Gauron T., Guzmán D., Hare T., Jang B.-H., Jang J.-G., Jordan A., Kim J., Kim K.M., de Oliveira C. M., The GMT-Consortium Large Earth Finder (G-CLEF): an optical Echelle spectrograph for the Giant Magellan Telescope (GMT), Proc. SPIE, 2016, vol. 9908

Szentgyorgyi A., Barnes S., Bean J., Bigelow B., Bouchez A., Chun M.-Y., Crane J. D., Epps H., Evans I., Evans J., Frebel A., Furesz G., Glenday A., Guzman D., Hare T., Jang B.-H., Jang J.-G., Jeong U., Jordan A., Kim K.-M., Kim J., Li C.-H., Lopez-Morales M., McCracken K., McLeod B., A preliminary design for the GMT-Consortium Large Earth Finder (G-CLEF), Proc. SPIE, 2014, vol. 9147

Szentgyorgyi A., Frebel A., Furesz G., Hertz E., Norton T., Bean J., Bergner H., Crane J., Evans J., Evans I., Gauron T., Jordán A., Park S., Uomoto A., Barnes S., Davis 
W., Eisenhower M., Epps H., Guzman D., McCracken K., Ordway M., Plummer D., Podgorski W., The GMT-CfA, Carnegie, Catolica, Chicago Large Earth Finder (G-CLEF): a general purpose optical echelle spectrograph for the GMT with precision radial velocity capability, Proc. SPIE, 2012, vol. 8446

Weiberg A., Birkby J., Bowler B., López-Molares M., Males J., Morzinski K., Wang S., Chapter 2: Exoplanets and Planet Formation In Giant Magellan Telescope Science Book 2018, 2018

Wolszczan A., Frail D. A., A planetary system around the millisecond pulsar PSR1257 + 12, Nature, 1992, vol. 355, p. 145 
Appendix 

Appendix A

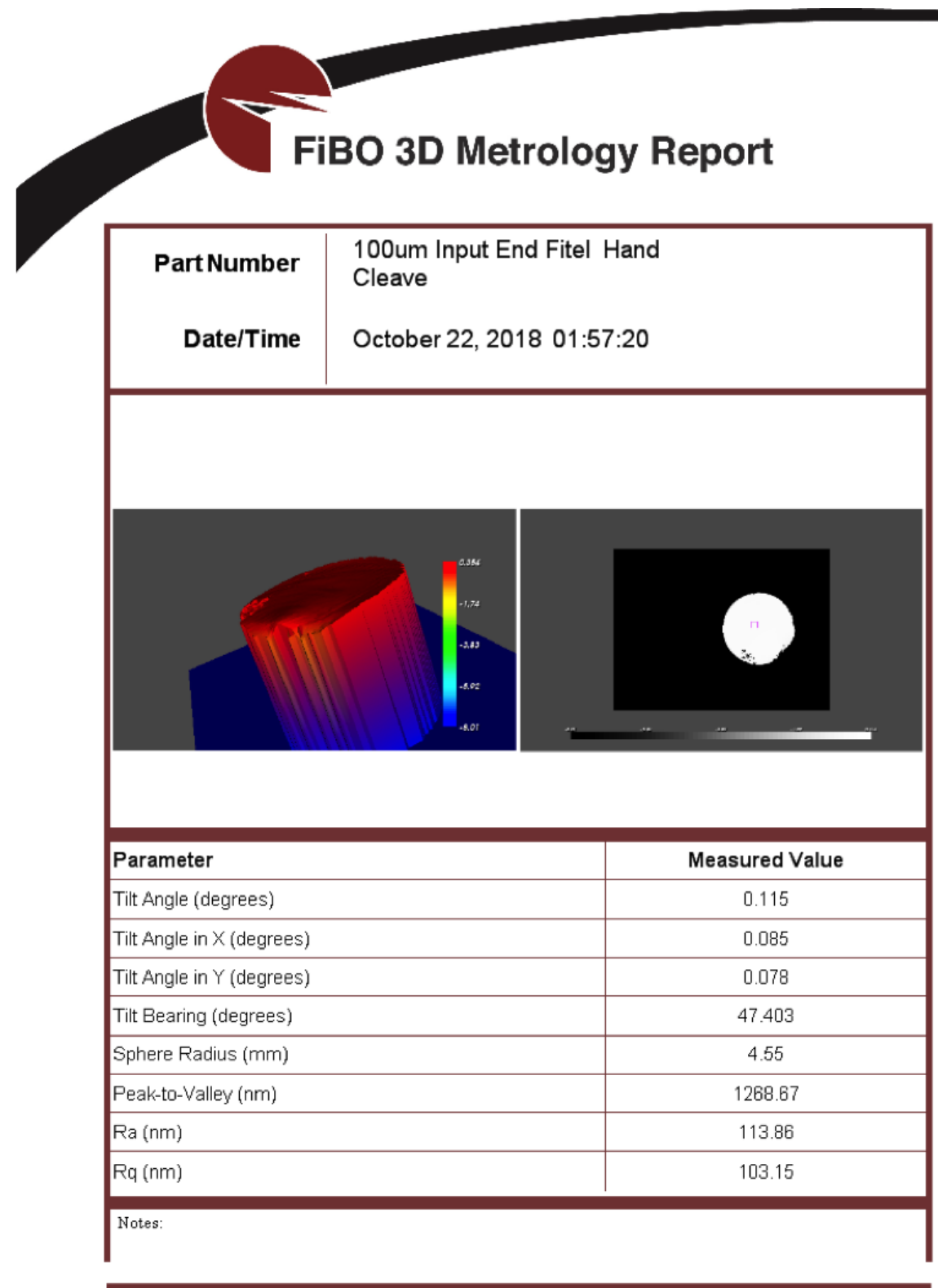

FiBO SN: 3530012

Figure A.1: Input fiber surface of 'Fiber 1', cleaved with LCC cleaver. This fiber was not used. 


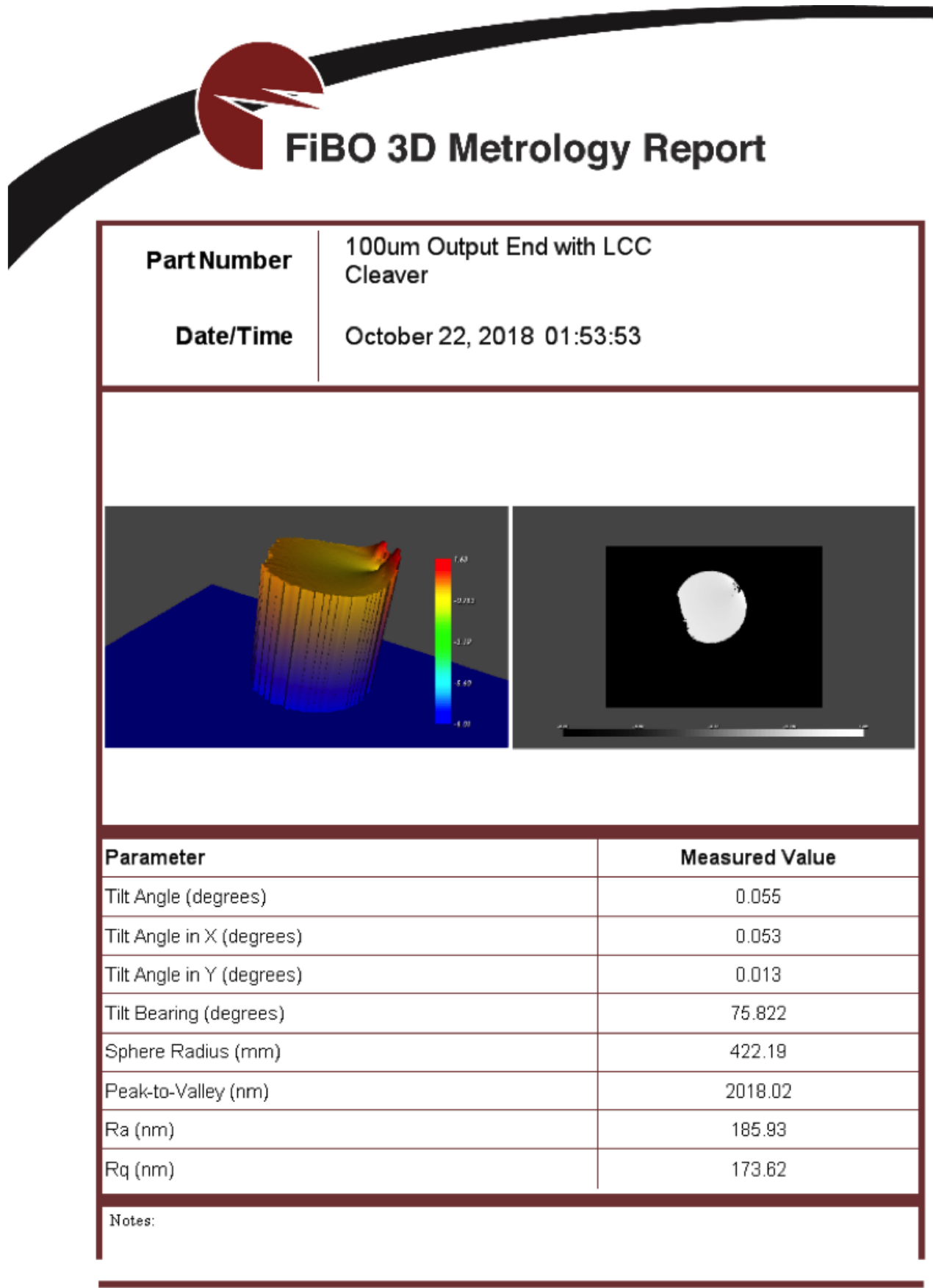

FiBO SN: 3530012

Figure A.2: Output fiber surface of 'Fiber 1', cleaved with LCC cleaver. This fiber was not used. 


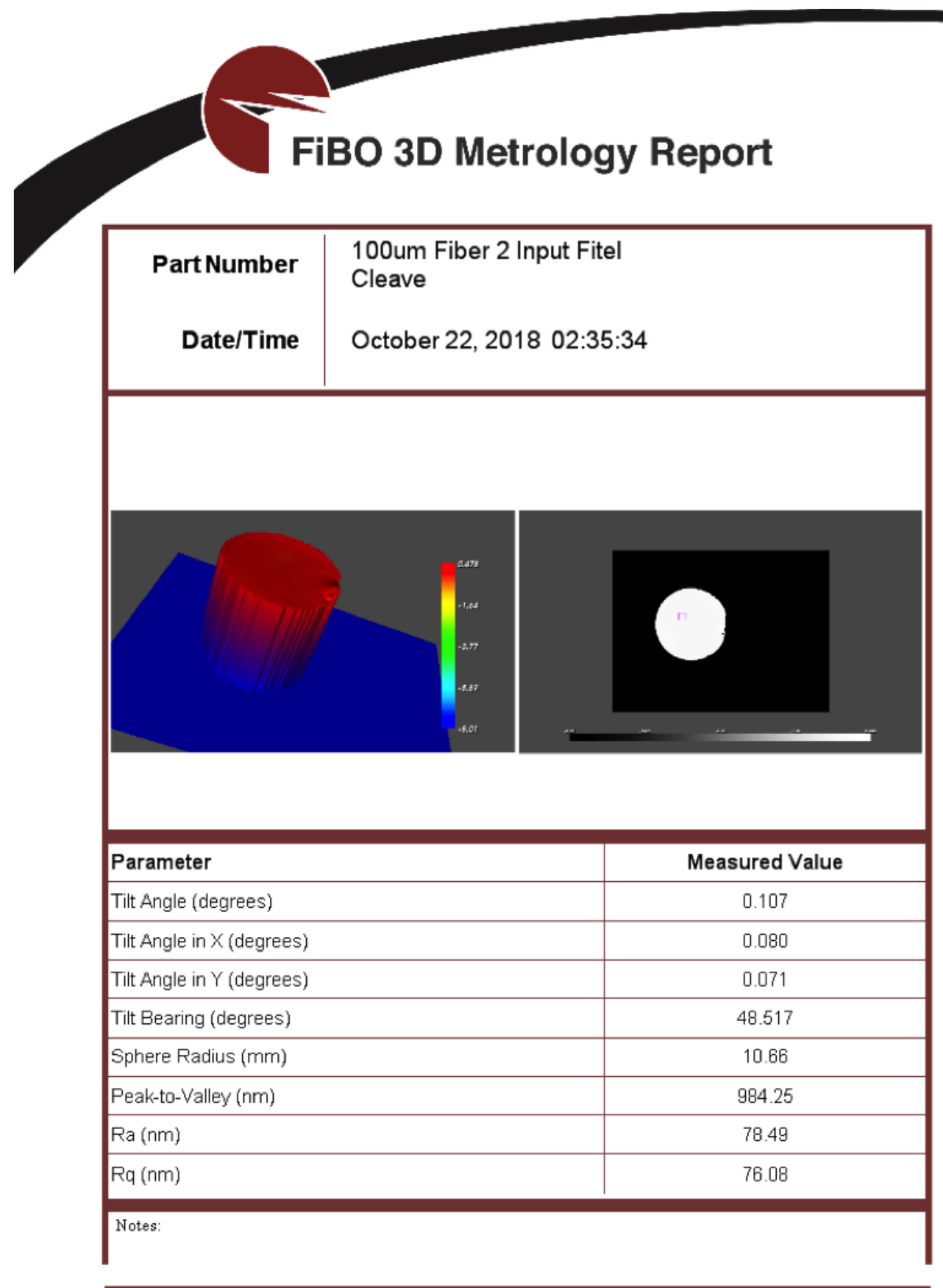

FiBO SN: 3530012

Figure A.3: Input fiber surface of 'Fiber 2', cleaved with common, commercial cleaver. This was the fiber used. 


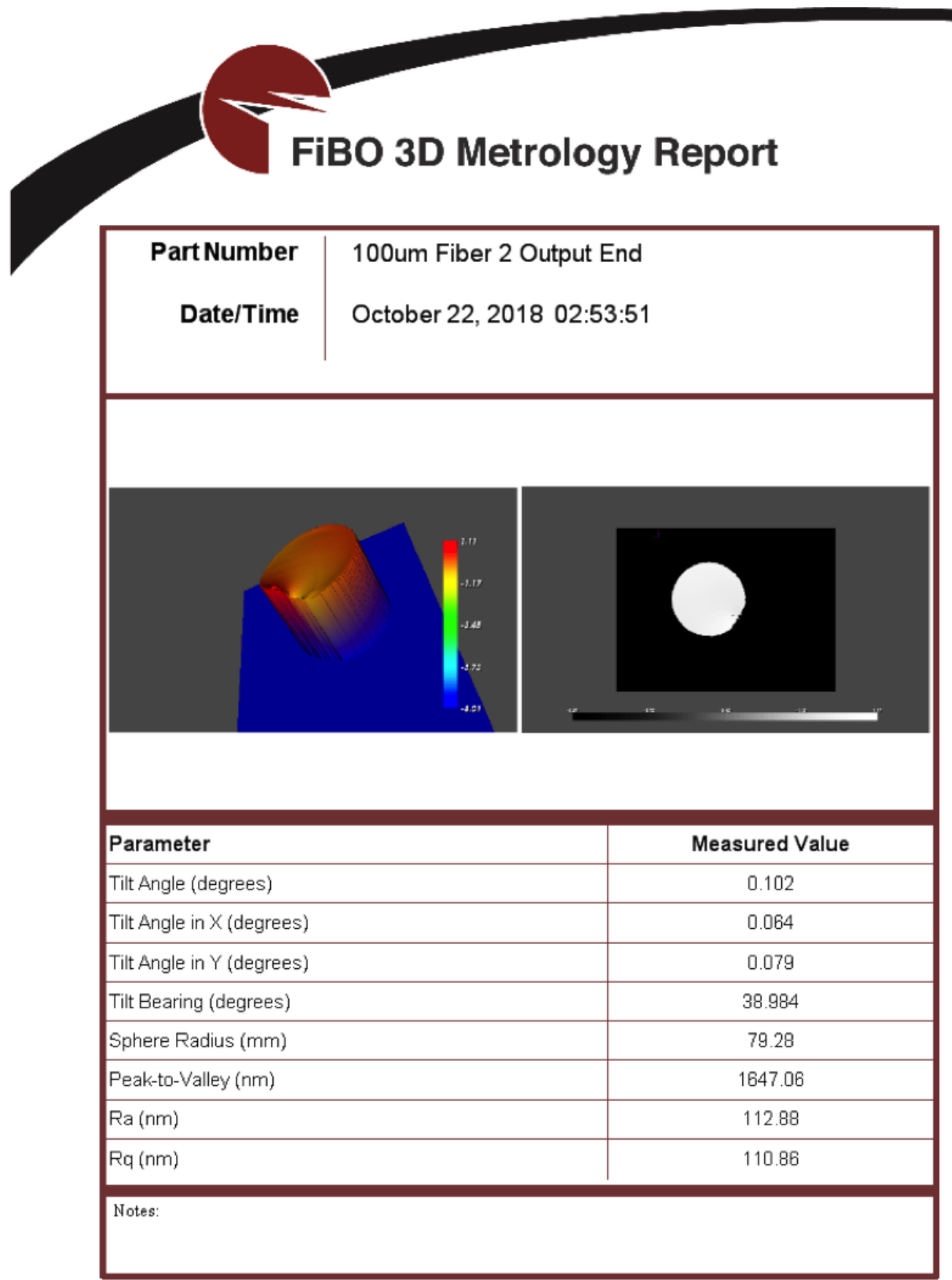

FiBO SN: 3530012

Figure A.4: Output fiber surface of 'Fiber 2', cleaved with common, commercial cleaver. This was the fiber used. 